\title{
The influence of matrix viscoelasticity on the rheology of polymer blends
}

\section{Journal Article}

\section{Author(s):}

Dressler, Marco; Edwards, Brian J.

Publication date:

2004-05

Permanent link:

https://doi.org/10.3929/ethz-b-000050746

Rights / license:

In Copyright - Non-Commercial Use Permitted

Originally published in:

Rheologica Acta 43(3), https://doi.org/10.1007/s00397-003-0341-8 


\section{Marco Dressler \\ Brian J. Edwards}

\section{The influence of matrix viscoelasticity on the rheology of polymer blends}

Received: 14 April 2003

Accepted: 29 October 2003

Published online: 29 January 2004

(c) Springer-Verlag 2004
M. Dressler $(\square)$

Institute of Food Science and Nutrition, Laboratory of Food Process Engineering, ETH-Zürich, CH-8092 Zürich, Switzerland E-mail: marco.dressler@ilw.agrl.ethz.ch

B. J. Edwards

Department of Chemical Engineering, The University of Tennessee Knoxville, Knoxville, TN 37996, USA

\begin{abstract}
We examine the effects of matrix phase viscoelasticity on the rheological modeling of polymer blends with a droplet morphology. Two contravariant, second-rank tensor variables are adopted along with the translational momentum density of the fluid to account for viscoelasticity of the matrix phase and the ellipsoidal droplet shapes. The first microstructural variable is a conformation tensor describing the average extension and orientation of the molecules in the matrix phase.

The other microstructural variable is a configuration tensor to account for the average shape and orientation of constant-volume droplets. A Hamiltonian framework of non-equilibrium thermodynamics is then adopted to derive a set of continuum equations for the system variables. This set of equations accounts for local conformational changes of the
\end{abstract}

matrix molecules due to droplet deformation and vice versa. The model is intended for dilute blends of both oblate and prolate droplets, and droplet breakup and coalescence are not taken into account. Only the matrix phase is considered as viscoelastic; i.e., the droplets are assumed to be Newtonian. The model equations are solved for various types of homogeneous deformations, and microstructure/rheology relationships are discussed for transient and steady-state conditions. A comparison with other constrained-volume rheological models and experimental data is made as well.

Keywords Non-equilibrium thermodynamics - Polymer blends · Constant-volume models · Rheological characterization . Oblate/prolate droplets

\section{Introduction}

Polymer blends are systems of technological importance since the blending of materials with specific properties is cheaper than the chemical synthesis of new polymers. From a scientific point of view, these systems are interesting since a set of generally accepted macroscopic time evolution equations to describe their complicated rheological response is not yet available. A main problem in the macroscopic description of polymer blends with a droplet morphology lies in the choice of the appropriate microstructural variable to account for the droplet shape. Two different second-rank tensor structural variables have been proposed in the literature thus far: a droplet shape tensor manifesting as a contravariant, second-rank structural tensor whose eigenvalues are the squared magnitudes of the droplet axes (Maffettone and Minale 1998), and an anisotropy tensor defined as a covariant, second-rank structural tensor which is the area average of the dyadic of the surface normal vector divided by the droplet volume (Almusallam et al. 2000; Doi and Ohta 1991). Following initial work on this 
subject by Doi and Ohta (1991), several attempts were undertaken recently to establish a more fundamental set of macroscopic time evolution equations for polymer blends, working either in terms of the droplet shape tensor (Maffettone and Minale 1998) or the anisotropy tensor (Almusallam et al. 2000). Recently, non-equilibrium thermodynamics has been used to obtain dynamically consistent time evolution equations for polymer blends with a droplet morphology and to propose expressions for the extra stress tensor that are thermodynamically consistent with the time evolution equation for the structural variable and the momentum balance (Aït-Kadi et al. 1999; Edwards and Dressler 2003; Edwards et al. 2003; Grmela et al. 2001).

A shortcoming of almost all theoretical works on polymer blend rheology, and in particular of the works dealing with the droplet shape tensor theory and the anisotropy tensor theory, is that they treat blends of Newtonian fluids, hence neglecting non-Newtonian flow behavior of the matrix fluid. Therefore, in these models the viscoelastic response of the blend is exclusively due to the elastic interface between the components. It is clear that the limitation of Newtonian blend components is a very severe one since commercial polymer blends are made of high molecular weight polymers with a characteristic non-Newtonian flow behavior. If the blend consists of a continuous matrix of one polymer with microscopic droplets of a second phase, the viscoelastic response is due to the matrix and not just the interface. Recently, Greco (2002) has treated the problem of drop deformation for non-Newtonian fluids in slow steady flows using a perturbative approach to find particular solutions of the relevant continuum equations. In the present work, we wish to propose a thermodynamic description for polymer blends consisting of a continuous phase with microscopically small droplet inclusions of a second phase, the former having a distinct viscoelastic flavor. We do this in order to explore the consequences that matrix phase non-Newtonian characteristics have on flow-induced droplet morphology, and to discern when such effects become relevant. In the next section we give a brief summary of relevant theoretical work, which, up to now, has been presented in the field of polymer blend rheology.

\section{Models for polymer blends with droplet morphology}

Over the past 15 years, non-equilibrium thermodynamics has become a well established theory with which to investigate fluids with internal microstructure (Beris and Edwards 1990a, 1990b, 1994; Edwards and Beris 1991a, Edwards et al. 1991; Grmela 1988, 1989; Grmela and Carreau 1987; Grmela and Öttinger 1997; Öttinger and Grmela 1997). Recently, polymer blends have become the subject of investigation in applied non-equilibrium thermodynamics, with the goal being to derive dynamical time evolution equations for these systems. The first attempts (Grmela and Aït-Kadi 1998; Grmela et al. 1998; Lacroix et al. 1998; Wagner et al. 1999) in this direction focused on blends of Newtonian liquids with a co-continuous morphology and, in particular, on the improvement of the Doi-Ohta (DO) Model (Doi and Ohta 1991). This model was developed for blends of equi-density and equi-viscosity Newtonian liquids. The research efforts of Grmela and Aït-Kadi (1998), Grmela et al. (1998), Lacroix et al. (1998), and Wagner et al. (1999) tried to obtain more realistic relaxation expressions within the context of the Doi-Ohta theory without changing the microstructural variable (the anisotropy tensor) of the original model. Recently, Lhuillier (2003) has examined coherently a blend of Newtonian fluids with unequal viscosities.

Since 1998, research activities in theoretical polymer blend rheology also shifted towards blends with a droplet morphology, where a disperse phase is present as microscopically small droplets in a continuous matrix. Maffettone and Minale (1998) introduced the first constrained-volume model for the deformation, orientation, and advection of ellipsoidal droplets of a Newtonian fluid in a second Newtonian matrix fluid. In this model, the droplet is described in terms of a second-rank, contravariant microstructural tensor with constant determinant to account for volume preservation of the disperse phase. The time evolution equation of the Maffettone-Minale (MM) Model is

$$
\begin{aligned}
\frac{\partial S_{\alpha \beta}}{\partial t}= & -v_{\gamma} \nabla_{\gamma} S_{\alpha \beta}+\frac{f_{2}+1}{2}\left(S_{\alpha \gamma} \nabla_{\gamma} v_{\beta}+S_{\beta \gamma} \nabla_{\gamma} v_{\alpha}\right) \\
& +\frac{f_{2}-1}{2}\left(S_{\alpha \gamma} \nabla_{\beta} v_{\gamma}+S_{\beta \gamma} \nabla_{\alpha} v_{\gamma}\right)-\frac{f_{1}}{\lambda}\left(S_{\alpha \beta}-\frac{3}{\operatorname{tr} S^{-1}} \delta_{\alpha \beta}\right)
\end{aligned}
$$

where $\mathbf{S}$ is the droplet configuration tensor, $\mathbf{v}$ is the velocity field, $\operatorname{tr}$ denotes the trace, $f_{1}, f_{2}$ are phenomenological parameters, and $\lambda$ is the relaxation time related to the interface between the two Newtonian fluids. In Eq. (1), an isotropic relaxation mechanism due to interfacial tension between the blend components is used implicitly to describe a viscoelastic recovery of the droplet phase and the concept of non-affine motion is adopted to recover non-axisymmetric droplet configurations with three different semiaxes. Note that the MM Model, as developed by Maffettone and Minale (1998), is purely phenomenological and does not give an expression for the extra stress tensor. Aït-Kadi et al. (1999) were the first researchers to undertake a thermodynamic study of general constrained-volume models which lead to a restricted set of time evolution equations for these systems. Later, this restricted set of time evolution equations was generalized by Edwards and Dressler (2003). Grmela et al. (2001) adopted a non- 
equilibrium thermodynamics approach and introduced a phenomenological expression for the elastic free energy to obtain a thermodynamically consistent expression for the extra stress tensor for the MM Model, which has been evaluated and compared with experimental data for emulsions by $\mathrm{Yu}$ et al. (2002a, 2002b).

An alternative constrained-volume model for blends of Newtonian liquids with a droplet morphology was presented in Almusallam et al. (2000). The AlmusallamLarson-Solomon (ALS) Model is written in terms of a modified anisotropy tensor of the DO type with nonvanishing trace, q. For this variable, an approximate volume for the droplet inclusions is defined in terms of the scalar invariants of $\mathbf{q}$, and a set of time evolution equations is proposed, which was intended to conserve the approximate volume related to the anisotropy tensor. However, this is a quite difficult task, which was fully accomplished only recently by Edwards and Dressler (2003) adopting the methodology proposed by Edwards et al. (2003).

The DO Model and the constrained-volume rheological models have been invoked in several instances (see, e.g., Jansseune et al. 2000, 2001) to recover morphological properties of polymer blends from rheological measurements. The idea of these works was to measure transient and steady-state shear stresses and first normal stress differences, which could be linked to an orientation angle via a stress-optical rule. Then, a rheological blend model such as the MM Model, the DO Model, or the ALS Model was invoked to obtain the average size of the dispersed droplets (Jansseune et al. 2000). A posteriori stress tensor expressions, such as the formula of Batchelor, have been adopted to obtain viscometric properties from morphological measurements - see, e.g., Minale and Maffettone (2003).

A common limitation of the DO Model, the MM Model, and the ALS Model, is that they make the assumption of Newtonian blend components. In the subsequent section, we wish to derive a set of continuum equations for polymer blends with a droplet morphology taking into account the non-Newtonian flow behavior of the viscoelastic matrix. We will develop our description in terms of the droplet shape tensor, $\mathbf{S}$, since it has a clear connection to the underlying spherical microstructure of the blend and it allows a rigorous, yet simple, representation of the droplet volume in terms of the determinant of the droplet shape tensor.

\section{Thermodynamic description of matrix-phase viscoelasticity in polymer blends}

In this section we develop a description for polymer blends with a droplet morphology that incorporates two second-rank structural tensor fields that are allowed to interact with each other under flow. The blend is modeled as a thermodynamic continuum with internal microstructure consisting of two immiscible phases. The thermodynamic variables in a continuum description of matter are statistical mechanical averages over all constituent molecules and other microstructural components which constitute a single fluid particle of the continuum. The average elongation and orientation of the matrix molecules at a fixed position in space is described in terms of a conformation tensor field. The shape and orientation of the droplets at a certain location in the continuum is accounted for in terms of a droplet shape tensor field, which can be viewed as a statistical mechanical average over the size and configuration of all droplets in a single fluid particle. The macroscopic flow of the blend is quantified in terms of the momentum density field, defined as the velocity of a fluid particle times its density. In the present work, we adopt the approximation of ellipsoidal droplet shapes with constant volume. For single droplets with diameters larger than microns $(40-500 \mu \mathrm{m})$, this approximation has been investigated experimentally by Guido and Villone (1998) and by $\mathrm{Hu}$ and Lips (2003) in simple shear flow and in planar hyperbolic flow, respectively. Implicitly, we assume the validity of the ellipsoidal droplet and constant-volume assumptions on smaller length scales than those examined experimentally. Furthermore, we neglect break-up and coalescence phenomena, this being a reasonable approximation in appropriate deformation and strain rate regimes. Note that the continuum equations to be derived in this section are not limited to a small deformation regime which is typically encountered in low amplitude oscillatory shear flow. In the subsequent section various sample calculations referring to the non-linear viscoelastic material behavior will be presented. We assume that the dispersed phase concentration and the material properties of the blend components are such that breakup and coalescence do not occur.

We wish to present only the most important steps in the construction of the relevant macroscopic flow equations since the basic ideas of the thermodynamic description of complex materials in terms of multiple conformation tensors are explained elsewhere (Beris and Edwards 1994; Edwards et al. 1996). The dynamical evolution equations for the polymer blend are obtained from a master equation

$\frac{\mathrm{d} F}{\mathrm{~d} t}=\{F, H\}+[F, H]$,

where $F=F[x]$ is an arbitrary functional of a set of field variables $x, H$ denotes the Hamiltonian or the generator of the dynamics, $\{\cdot, \cdot\},[\cdot, \cdot]$ denote the Poisson and dissipation brackets, respectively, and $\mathrm{d} \cdot / \mathrm{d} t$ is the time derivative. Equation (2) is a special case of the more 
general GENERIC expression of Grmela and Öttinger (1997) and Öttinger and Grmela (1997), applied herein to isothermal and incompressible fluids. The physical variables for the description of the blend are the momentum density, $\mathbf{M}=\rho \mathbf{v}$ ( $\rho$ being the effective mass density of the medium and $\mathbf{v}$ the velocity field), an unconstrained contravariant second-rank tensor, C, describing the microstructure of the continuous phase, i.e., the conformation of the polymer molecules in the matrix, and a constrained contravariant second-rank tensor, $\mathbf{S}$, to describe ellipsoidal droplet shapes: hence $x=[\mathbf{M}, \mathbf{C}, \mathbf{S}]$. Since the disperse phase is assumed to be incompressible, we impose the microstructural constraint, $\operatorname{det} \mathbf{S}=1$, to account for volume preservation of the deforming micro-droplets. Further droplet configuration tensors or conformation tensors may be included into the above set of variables to describe systems with non-uniform droplet size distribution or rheologically more complex matrix fluids, if desired.

To derive a set of continuum equations using Eq. (2), one has to specify the Poisson and dissipation brackets, as well as the generator of the dynamics. The Poisson bracket for a viscoelastic fluid described in terms of $x$ has been derived in Beris and Edwards (1990a, 1990b, 1994), Edwards and Beris (1991a, 1991b), and Edwards et al. (1991, 2003). For the dissipation bracket, we adopt the following expression:

$$
\begin{aligned}
{[F, H]=} & -\int \Lambda_{\alpha \beta \gamma \in}^{\mathrm{C}} \frac{\delta F \delta H}{\delta C_{\alpha \beta} \delta C_{\gamma \in}} \mathrm{d}^{3} x-\int \Lambda_{\alpha \beta \gamma}^{\mathrm{S}} \frac{\delta F \delta H}{\delta S_{\alpha \beta} \delta S_{\gamma \in}} \mathrm{d}^{3} x \\
& +\frac{1}{3} \int \Lambda_{\alpha \beta \gamma \in}^{\mathrm{S}} S_{\rho \eta} S_{\alpha \beta}^{-1} \frac{\delta F \delta H}{\delta S_{\rho \eta} \delta S_{\gamma \in}} \mathrm{d}^{3} x \\
& -\int A_{\alpha \beta \gamma \in}\left(\frac{\delta F \delta H}{\delta C_{\alpha \beta} \delta S_{\gamma \in}}+\frac{\delta H}{\delta C_{\alpha \beta} \delta S_{\gamma \in}}\right) \mathrm{d}^{3} x \\
& +\frac{1}{3} \int A_{\alpha \beta \gamma \in}\left(\frac{\delta F \delta H}{\delta C_{\alpha \beta} \delta S_{\rho \eta}} S_{\rho \eta} S_{\gamma \in}^{-1}+\frac{\delta H \delta F}{\delta C_{\alpha \beta} \delta S_{\rho \eta}} S_{\rho \eta} S_{\gamma \in}^{-1}\right) \mathrm{d}^{3} x,
\end{aligned}
$$

with the three phenomenological matrices $\Lambda^{\mathrm{C}}, \Lambda^{\mathrm{S}}$, and A. The above dissipation bracket represents a generalization of the dissipation bracket for the Two Coupled Maxwell Modes Model of Beris and Edwards (1994) to a system that is described in terms of an unconstrained conformation tensor, $\mathbf{C}$, and a constrained droplet tensor, $\mathbf{S}$, with $\operatorname{det} \mathbf{S}=1$. The first integral in the above dissipation bracket is the relaxation of the viscoelastic matrix. The second and the third integral account for the relaxation of the droplet inclusions under the auspices of the constraint $\operatorname{det} \mathbf{S}=1$, and were derived by Edwards et al. (2003). The last two integrals, involving the phenomenological matrix A, describe the coupling of the viscoelastic matrix fluid with the droplet interface and they have been derived with the procedure developed in Edwards et al. (2003). In the above dissipation bracket, we neglect viscous dissipation of the matrix fluid since it appears implicitly in the Maxwell viscosity $\left(n_{\mathrm{C}} k_{\mathrm{B}} T \lambda_{\mathrm{C}}\right.$ - see below for definitions of these symbols if unclear) and droplet diffusivity. The latter phenomenon can be included in the dissipation bracket to obtain a more sophisticated set of equations for this system, if so desired.

The Poisson and dissipation brackets yield a set of dynamical evolution equations of the form

$$
\begin{aligned}
& \rho \frac{\partial v_{\alpha}}{\partial t}=-\rho v_{\beta} \nabla_{\beta} v_{\alpha}-\nabla_{\alpha} p+\nabla_{\beta} \sigma_{\alpha \beta}, \\
& \frac{\partial C_{\alpha \beta}}{\partial t}=\left.\frac{\partial C_{\alpha \beta}}{\partial t}\right|_{\text {cons }}+\left.\frac{\partial C_{\alpha \beta}}{\partial t}\right|_{\text {diss }}, \\
& \frac{\partial S_{\alpha \beta}}{\partial t}=\left.\frac{\partial S_{\alpha \beta}}{\partial t}\right|_{\text {cons }}+\left.\frac{\partial S_{\alpha \beta}}{\partial t}\right|_{\text {diss }} .
\end{aligned}
$$

Equation (4a) is the momentum balance equation in a spatial description of macroscopic fluid flow, where the pressure and the extra stress tensor have been denoted with $p$ and $\sigma$, respectively. Pressure and velocity are thus viewed as averaged quantities at each location in spacetime coordinates; i.e., they are coarse-grained averages of the matrix fluid particles and droplets contained in the fluid particle at $(\mathbf{x}, t)$. They arise naturally through the mathematical structure of the Poisson bracket. The pressure obeys a Poisson equation with appropriate boundary conditions, and the extra stress tensor is derived as

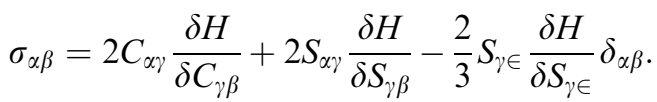

Equations (4b) and (4c) are the time evolution equations for the structural tensors, each with a conservative and a dissipative part. The conservative terms in the time evolution equations are given as

$$
\begin{aligned}
& \left.\frac{\partial C_{\alpha \beta}}{\partial t}\right|_{\text {cons }}=-v_{\gamma} \nabla_{\gamma} C_{\alpha \beta}+C_{\alpha \gamma} \nabla_{\gamma} v_{\beta}+C_{\beta \gamma} \nabla_{\gamma} v_{\alpha} \\
& \left.\frac{\partial S_{\alpha \beta}}{\partial t}\right|_{\text {cons }}=-v_{\gamma} \nabla_{\gamma} S_{\alpha \beta}-\frac{2}{3} \nabla_{\gamma} v_{\gamma} S_{\alpha \beta}+S_{\alpha \gamma} \nabla_{\gamma} v_{\beta}+S_{\beta \gamma} \nabla_{\gamma} v_{\alpha}
\end{aligned}
$$

Equation (6a) is the upper-convected derivative of the second-rank tensor, C, and Eq. (6b) is the corresponding derivative for a tensor with the determinant constrained to unity. The dissipative contributions to Eqs. (4b) and (4c) follow from the dissipation bracket, Eq. (3): 


$$
\begin{aligned}
& \left.\frac{\partial C_{\alpha \beta}}{\partial t}\right|_{\text {diss }} \\
& =-\Lambda_{\alpha \beta \gamma \in}^{\mathrm{C}} \frac{\delta H}{\delta C_{\gamma \in}}-A_{\alpha \beta \gamma \in} \frac{\delta H}{\delta S_{\gamma \in}}+\frac{1}{3} A_{\alpha \beta \gamma \in} \frac{\delta H}{\delta S_{\rho v}} S_{\rho v} S_{\gamma \in}^{-1}, \\
& \begin{aligned}
\left.\frac{\partial S_{\alpha \beta}}{\partial t}\right|_{\text {diss }}= & -\Lambda_{\alpha \beta \gamma \in \in}^{\mathrm{S}} \frac{\delta H}{\delta S_{\gamma \epsilon}}+\frac{1}{3} \Lambda_{\rho \eta \gamma \in}^{\mathrm{S}} S_{\alpha \beta} S_{\rho \eta}^{-1} \frac{\delta H}{\delta S_{\gamma \epsilon}}-A_{\alpha \beta \gamma \in \frac{\delta H}{\delta C_{\gamma \epsilon}}} \\
& +\frac{1}{3} A_{\rho v \gamma \in \frac{\delta H}{\delta C_{\rho v}}} S_{\gamma \in}^{-1} S_{\alpha \beta} .
\end{aligned}
\end{aligned}
$$

With the procedure developed by Edwards et al. (2003), it can be shown for Eqs. (6b) and (7b) that detS is a conserved quantity independent of the mathematical form of the phenomenological matrices $\Lambda^{\mathrm{C}}, \Lambda^{\mathrm{S}}, \mathbf{A}$, and the Hamiltonian, $H[\mathbf{M}, \mathbf{C}, \mathbf{S}]$. In order to obtain a specific set of system equations from the set of Eqs. $(4,4 b, 4 c)$, we have to define expressions for the Hamiltonian and the phenomenological matrices appearing in the dissipation bracket, Eq. (3). These are the four ingredients required to reduce the set of general time evolution equations (Eqs. 4a, 4b, 4c, 5, 6a, 6b, 7a and 7b) to a specific model for the polymer blend.

In what follows, we wish to study a system which can be envisioned as a Maxwell fluid with characteristic elastic constant, $K$, coupling non-linearly to an elastic interface with interfacial tension, $\Gamma^{\prime}$. One particular realization of such a system can be described in terms of the Hamiltonian

$$
\begin{aligned}
H_{m}[M, C, S]=K[M]+A[C, S] \\
=\int\left[\frac{M_{\alpha} M_{\alpha}}{2 \rho}+\frac{1}{2} n_{\mathrm{C}} K(1-\phi) \operatorname{tr} C\right. \\
\left.\quad-\frac{1}{2} n_{\mathrm{C}} k_{\mathrm{B}} T(1-\phi) \ln (\operatorname{det} C)+\frac{1}{2} \Gamma \phi I_{2}^{\mathrm{S}}\right] \mathrm{d}^{3} x,
\end{aligned}
$$

where $I_{2}^{\mathrm{S}}$ is the second invariant of $\mathbf{S}, \phi$ is the concentration of droplets, $n_{\mathrm{C}}$ is the degree of elasticity per unit volume of the matrix, and $\Gamma=\Gamma^{\prime} / R$ where $R$ is the average droplet radius in the undeformed state. Equation (8) represents the kinetic energy of the system (first term in the integral) plus a linear superposition of the elastic free energy of a system of Hookean springs (second and third terms) and the energy of the elastic interface, with $I_{2}^{\mathrm{S}}$ being associated with the droplet surface area (fourth term). The subscript " $\mathrm{m}$ " in the Hamiltonian denotes that the description of the polymer blend is purely mechanical, i.e., we have not considered a balance equation for the entropy density to account for the transfer of mechanical energy into internal degrees of freedom. Note that the last term in the integral of Eq. (8) is different from the expression for the thermodynamic potential introduced in Grmela et al. (2001).
A physical justification for the choice for this term is that the specific surface energy is proportional to the surface area (at least for small deviations from sphericity) and it assumes a minimum for the spherical droplet.

Next, we specify the dissipative phenomenological coefficients appearing in the bracket of Eq. (3). We adopt the phenomenological matrix for the Maxwell Model to describe the relaxation of the matrix fluid (Beris and Edwards 1994):

$\Lambda_{\alpha \beta \gamma \in}^{\mathrm{C}}=\frac{1}{2 G \lambda_{\mathrm{C}}} \frac{k_{\mathrm{B}} T}{K}\left(C_{\alpha \gamma} \delta_{\beta \in}+C_{\alpha \in} \delta_{\beta \gamma}+C_{\beta \gamma} \delta_{\alpha \in}+C_{\beta \in} \delta_{\alpha \gamma}\right)$,

where $\lambda_{\mathrm{C}}$ is a characteristic relaxation time associated with the continuous phase and we have introduced the elastic modulus of the continuous phase $G=n_{\mathrm{C}} k_{\mathrm{B}} T$. In conjunction with the $\mathbf{C}$-terms in the above Hamiltonian, Eq. (8), and with $\mathbf{A}=0$, this expression gives the UpperConvected Maxwell Model (UCMM) for the matrix phase. To fit the model to experimental data it is necessary to incorporate the viscoelastic characteristics of the matrix phase into the relaxation matrix $\Lambda_{C}$. Here we use a variable relaxation time, $\lambda_{\mathrm{c}} \equiv \lambda_{\mathrm{c}}(1 / 3 \operatorname{tr} \tilde{C})^{k}(k$ being a power law index, $\left.\tilde{C}=C K / k_{\mathrm{B}} T\right)$, according to the Extended White-Metzner (EWM) Model of Souvaliotis and Beris (1992) to incorporate the effects of matrix shear thinning into the model. Furthermore, we adopt the following anisotropic expression for the relaxation matrix of the interface:

$$
\begin{aligned}
\Lambda_{\alpha \beta \gamma \in}^{\mathrm{S}}= & \frac{1}{\Gamma \lambda_{\mathrm{S}}}\left[\frac{(1+p)^{2} p}{2}\left(S_{\alpha \gamma} \delta_{\beta \in}+S_{\alpha \in} \delta_{\beta \gamma}+S_{\beta \gamma} \delta_{\alpha \in}+S_{\beta \in} \delta_{\alpha \gamma}\right)\right. \\
& \left.+\frac{3 p}{I_{1}^{\mathrm{S}} I_{2}^{\mathrm{S}}}\left(\delta_{\alpha \gamma} \delta_{\beta \in}+\delta_{\alpha \in} \delta_{\beta \gamma}\right)\right]
\end{aligned}
$$

where $\lambda_{\mathrm{S}}$ is a characteristic time scale associated with the elastic interface and $I_{1}^{\mathrm{S}}$ is the first invariant of $\mathbf{S}$. The quantity $p$ is a phenomenological parameter which depends on the viscoelastic properties of the phases and will be specified below. The first term in the square brackets accounts for droplets which deform into oblates for startup of steady shearing flow, the second term accounts for droplets which deform into prolates for startup of steady shearing flow. The difference between oblate and prolate droplet shapes is illustrated in subsequent figures, which will be explained in more detail in the next section.

The phenomenological matrix $\boldsymbol{A}$ is adopted in analogy with the coupling matrix introduced by Beris and Edwards (1994):

$$
A_{\alpha \beta \gamma \in}=\frac{1}{2} \frac{(1+p)^{2} \theta}{\sqrt{G \Gamma \lambda_{C} \lambda_{\mathrm{S}}}}\left(C_{\alpha \gamma} S_{\beta \in}+C_{\alpha \in} S_{\beta \gamma}+C_{\beta \gamma} S_{\alpha \in}+C_{\beta \in} S_{\alpha \gamma}\right),
$$


where $\theta$ is a phenomenological coupling parameter. This relaxation matrix has to be generalized in analogy to Eq. (9) if the shear thinning behavior of the matrix phase is incorporated into the modeling, i.e. $\lambda_{\mathrm{C}} \equiv \lambda_{\mathrm{C}}(1 / 3 \operatorname{tr} \tilde{C})^{k}$. For $\theta>0$, the above phenomenological matrix gives oblate droplet configurations. Note that the coupling matrix vanishes for $p=-1$. The phenomenological coefficient $\theta$ may be taken as a function of the scalar invariants of the structural variables, if so desired; however, here we want to work with $\theta$ being a constant, real number for simplicity. There is no universal recipe available to determine the phenomenological parameter, $\theta$, and we propose how this coefficient could be related either to morphological or viscometric properties of the blend: If morphological properties of the blend can be measured easily in a well-defined flow field (e.g., in steady shear flow) then one might study the corresponding solutions of the system equations as a function of $\theta$ and use the value for $\theta$ that gives the most satisfactory fit to experimental data. If morphological properties cannot be measured easily, then one might refer to nonlinear viscometric properties of the blend and to calculate these properties as a function of the coupling parameter from the time evolution equations. This method has been proposed in Edwards et al. (1996) where the negative ratio of the normal stress differences has been chosen as the viscometric property from which to obtain a value for the coupling parameter.

In order to rationalize the thermodynamic admissibility of the phenomenological matrices (Eqs. 9, 10, and 11 , we consider the rate of mechanical energy dissipation generated by the Hamiltonian, Eq. (8):

$\frac{\mathrm{d} H_{\mathrm{m}}}{\mathrm{d} t}=\left[H_{m}, H_{m}\right] \leqslant 0$,

which is a decreasing function of time (Beris and Edwards 1994). Equation (12) is obtained from Eq. (2) by exploiting the antisymmetry of the Poisson bracket and the fact that mechanical energy has to be dissipated into internal degrees of freedom in the long time limit. Inequality (12) is the appropriate condition to give a physically meaningful description of the system, and it can lead to counterintuitive results for the range of thermodynamically admissible phenomenological coefficients adopted herein. Mathematical criteria which are imposed directly onto the dissipative phenomenological coefficients, e.g., on the relaxation times, $\lambda_{\mathrm{C}}$ and $\lambda_{\mathrm{S}}$, on the coupling parameter, $\theta$, or on the dissipative coefficient, $p$, do not necessarily satisfy the inequality (12), and may therefore lead to aphysical results. This will become evident in the following paragraph, where we notice that the dissipative phenomenological coefficient, $p$, has to be negative to account for a physically meaningful droplet relaxation according to the phenomenological matrix of Eq. (10).
For the dissipation bracket of Eq. (3), the inequality (Eq. 12) is equivalent to

$$
\begin{aligned}
{\left[H_{m}, H_{m}\right]=} & {\left[H_{m}, H_{m}\right]_{\mathrm{C}}+\left[H_{m}, H_{m}\right]_{\mathrm{S}}+\left[H_{m}, H_{m}\right]_{\mathrm{CS}} } \\
= & -\frac{(1-\phi)^{2} G}{2 \lambda_{\mathrm{C}}} \int\left(\frac{K}{k_{\mathrm{B}} T} I_{1}^{C}+\frac{k_{\mathrm{B}} T}{K} I_{-1}^{\mathrm{C}}-6\right) \mathrm{d}^{3} x \\
& -\frac{(1+p)^{2} p \phi^{2} \Gamma}{2 \lambda_{\mathrm{S}}} \int\left(3-\frac{1}{3} I_{1}^{\mathrm{S}} I_{2}^{\mathrm{S}}\right) \mathrm{d}^{3} x-\frac{p \phi^{2} \Gamma}{\lambda_{\mathrm{S}}} \\
& \times \int I_{2}^{\mathrm{S}}\left(3 \frac{I_{1}^{\mathrm{S}}}{\left(I_{2}^{\mathrm{S}}\right)^{2}}-1\right) \mathrm{d}^{3} x \\
& -\frac{(1+p)^{2} \phi(1-\phi) \theta \sqrt{G \Gamma} \frac{K}{k_{\mathrm{B}} T}}{\sqrt{\lambda_{\mathrm{C}} \lambda_{\mathrm{S}}}} \\
& \times \int\left(I_{1}^{\mathrm{S}} C_{\alpha \beta} S_{\alpha \beta}-C_{\alpha \gamma} S_{\beta \gamma} S_{\alpha \beta}-\frac{2}{3} I_{1}^{\mathrm{C}} I_{2}^{\mathrm{S}}\right) \mathrm{d}^{3} x \leqslant 0,
\end{aligned}
$$

where $I_{-1}^{\mathrm{C}}=C_{\alpha \alpha}^{-1}=I_{2}^{\mathrm{C}} / I_{3}^{\mathrm{C}}$. The first integral is the mechanical energy dissipation of a Maxwell fluid, $\left[H_{\mathrm{m}}, H_{\mathrm{m}}\right]_{\mathrm{C}}$, the second and third integrals are the mechanical energy dissipation due to droplet relaxation including oblate and prolate relaxation, $\left[H_{\mathrm{m}}, H_{\mathrm{m}}\right]_{\mathrm{s}}$, and the fourth integral is the rate of mechanical energy dissipation due to the irreversible coupling of the matrix fluid and the droplet interface, $\left[H_{\mathrm{m}}, H_{\mathrm{m}}\right]_{\mathrm{CS}}$. Each of the first three integrals on the right-hand side of Eq. (13) has to be negative since they account for matrix chain relaxation and droplet retraction, respectively. This specifies the range of thermodynamically admissible phenomenological coefficients, $\lambda_{\mathrm{C}}, \lambda_{\mathrm{S}}, p$. Furthermore, the last integral has to be negative since the irreversible coupling between the two phases should not lead to an increase of mechanical energy in the long time limit. If one of the four integrals was positive, the mechanical dissipation rate could become positive for specific values of the physical variables.

In the following, we want to evaluate generically the four integrals in Eq. (13) for start-up of homogeneous, weak shear flow, $\dot{\gamma}<<1$, and for vanishing coupling parameter, $\theta=0$, to find the correct range of the thermodynamically admissible phenomenological coefficient p. Numerical calculations (cf. Fig. 1) corroborate that the scalar invariants of the structural variables increase upon start-up of steady shear flow (except the third invariant of the droplet shape tensor, $\operatorname{det} \mathbf{S}=1)$. The invariant $I_{-1}^{\mathrm{C}}$ assumes values above its equilibrium value, $I_{-1}^{\mathrm{C}}=3 K /\left(k_{\mathrm{B}} T\right)$; however, the variations in $I_{-1}^{\mathrm{C}}$ are small compared to the variations of $I_{1}^{\mathrm{C}}$. Therefore, the first integral in Eq. (13) is negative since the expression in parentheses is positive (the relaxation times, $\lambda_{\mathrm{C}}, \lambda_{\mathrm{S}}$, and the elastic moduli, $G, \Gamma$, are positive numbers). Due to $I_{1}^{\mathrm{S}} \geqslant 3$ and $I_{2}^{\mathrm{S}} \geqslant 3$, the expressions in parentheses in the second and the third integrals are negative. Conse- 
Fig. 1 The invariants of the structural variables, $\mathbf{C}$ and $\mathbf{S}$, and the mechanical dissipation steady shear flow, $\dot{\gamma}=0.1$, as a function of strain, $\gamma$. The parameters in the model have been specified as follows: $G /$ $\Gamma=4$ for the ratio of elastic strengths, $\lambda_{\mathrm{C}} / \lambda_{\mathrm{S}}=1$ for the ratio of relaxation times (i.e., $p=-0.25$ ), and $\phi=0.1$ for the dispersed phase concentration. We chose two values for the coupling parameter, $\theta=0$ (solid lines) and 0.1 (dashed lines) rate, $\left[H_{\mathrm{m}}, H_{\mathrm{m}}\right] / \Omega$ for start-up of
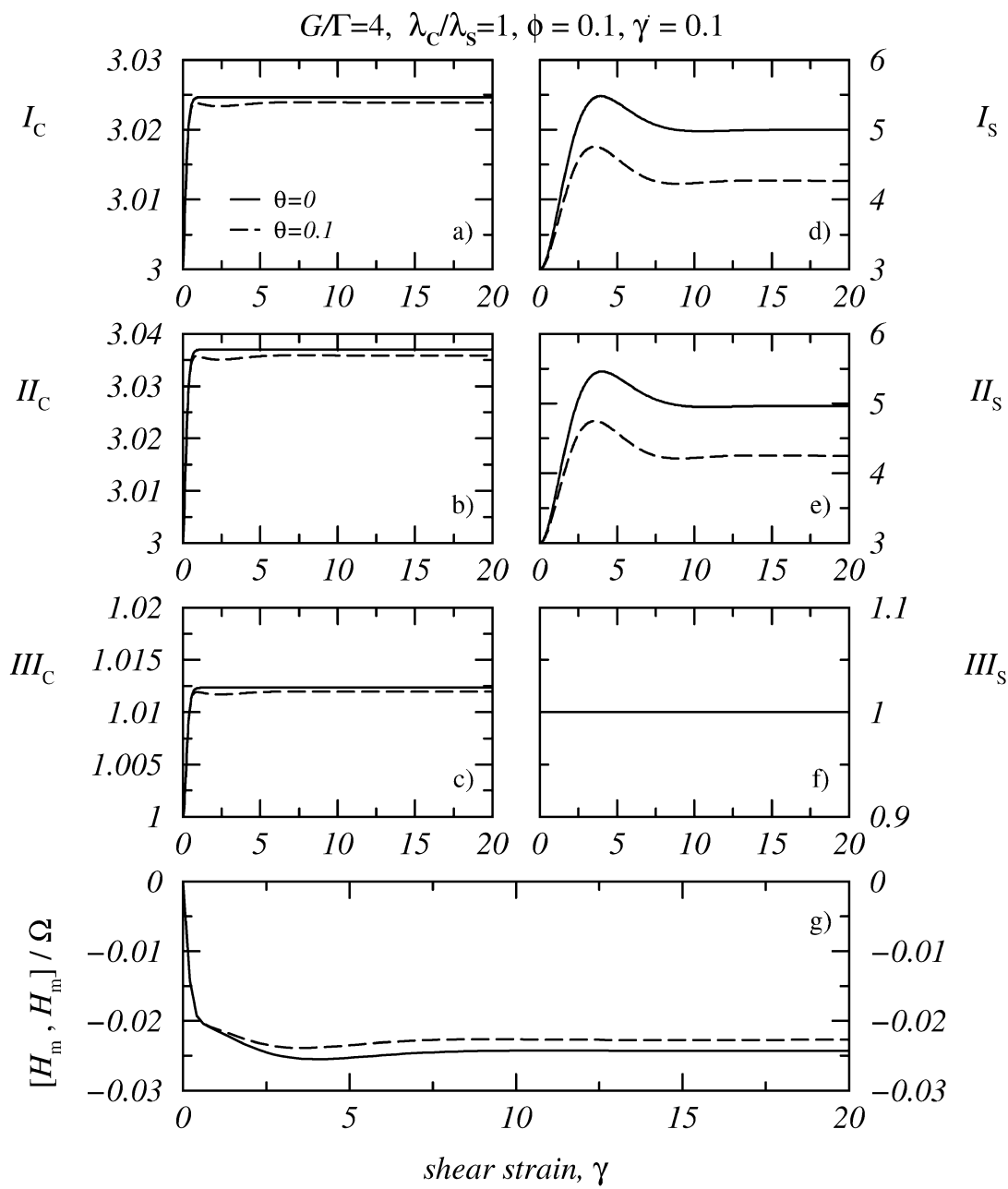

quently, the phenomenological friction coefficient, $p$, has to be negative to give a negative dissipation rate due to droplet relaxation.

Neglecting the prolate contribution in Eq. (10) for the time being (but still keeping $\theta=0$ ), we notice that the range of thermodynamically admissible phenomenological coefficients $\lambda_{\mathrm{C}}, \lambda_{\mathrm{S}}$, and $p$ in the two relaxation matrices at Eqs. (9) and (10) is different, although both expressions have an identical mathematical form. The deduction of the thermodynamic admissibility criterion $p<0$ from Eq. (10), arguing that the droplets relax to the lowest energy state of the spherical droplet in absence of entropic forces and flow, is not obvious. Instead the admissibility criterion $p<0$ is obtained from Eq. (12). Our analysis shows that the constraint $\operatorname{det} \mathbf{S}=1$ and the functional form of the thermodynamic potential, $E_{S}=1 / 2 \Gamma \phi I_{2}^{\mathrm{S}}$, produce this counterintuitive example of a phenomenological dissipative coefficient being negative. Nevertheless, if $p$ is taken as positive, then there is a direct violation of the Second Law of Thermodynamics.

For a vanishing coupling parameter, $\theta=0$, the last integral in Eq. (12) is zero. For a small value of the coupling parameter, $\theta<<1$, the coupling parameter has to be positive to yield a negative dissipation of mechanical energy due to the coupling between the two variables. Note that the possibility of a negative phenomenological friction coefficient was also found in Edwards et al. (1996), where the coupling parameter was shown to be $\theta \in[-1,1]$. Furthermore, note that the aspect of a negative friction coefficient in the droplet configuration equation is not worked out rigorously by Grmela et al. (2001) and Yu et al. (2002a).

Since experimental evidence suggests that the deformation behavior of droplets in a continuous phase into prolates and oblates (see, e.g., Guido and Villone 1998; Levitt et al. 1996) depends on the viscosity ratio and the linear viscoelastic properties of the blend components, we take $p$ as the negative viscosity ratio, $p=-\eta_{\mathrm{d}} / \eta_{\mathrm{c}}$. If the viscosity of the interface is considered the only relevant quantity to characterize the droplet phase and it is taken as a viscosity defined in analogy with the Maxwell Model, $\eta_{\mathrm{d}}=\Gamma \lambda_{\mathrm{S}}$, then the viscosity ratio, $p$, is not an independent quantity since we have $p=-\left(\Gamma \lambda_{\mathrm{S}}\right) /\left(G \lambda_{\mathrm{C}}\right)$, which is determined once the linear viscoelastic 
properties of the matrix and the interface are defined. We clarify that the quantity $\eta_{\mathrm{d}}$ is not the Newtonian viscosity of the droplet phase but it is the Maxwellian viscosity which is related to the interfacial elastic modulus, $\Gamma$, and the droplet relaxation time, $\lambda_{\mathrm{s}}$.

In what follows, we motivate this choice of the relevant physical parameter to describe droplet deformation in blends with matrix phase viscoelasticity and we provide a clarification concerning the effects of the matrix phase on the dynamics of droplets as compared to blends of Newtonian liquids. For blends of Newtonian liquids the droplet dynamics is governed by two dimensionless groups: the viscosity ratio and the Capillary number. For the present study we assume that droplet deformation and retraction are only due to interfacial tension between the two phases since there is no secured knowledge on the physical mechanisms of droplet dynamics in blends of non-Newtonian liquids. Therefore, the elastic free energy in Eq. (8) has been chosen to depend on the surface area of the droplets, $I_{S}^{2}$, and the relaxation matrix of Eq. (10) accounts for viscoelasticity of the interface. It will become evident in the next section when we render the equations dimensionless that this means a reduction of the number of dimensionless groups since the Capillary Number becomes the negative inverse of the viscosity ratio. However, it is also possible to introduce explicitly the Newtonian viscosity of the droplet phase, $\eta^{*}$, along with $\eta_{\mathrm{c}}=G \lambda_{\mathrm{C}}$ but instead of $\eta_{\mathrm{d}}=\Gamma \lambda_{\mathrm{s}}$. In this case $p=-\eta^{*} / \eta_{\mathrm{c}}$ becomes the negative ratio of the Newtonian viscosities of the blend components, and droplet dynamics are governed by the viscosity ratio, $p$, and interfacial modulus, $\Gamma$, as known from blends of Newtonian liquids.

Evaluating the Volterra derivatives of the Hamiltonian (8) and inserting the phenomenological matrices of Eqs. (9) and (10) into Eqs. (7a and 7b), we obtain the dissipative contributions to the time evolution equations of the structural variables:

$$
\begin{aligned}
\left.\frac{\partial C_{\alpha \beta}}{\partial t}\right|_{\text {diss }}= & -\frac{1-\phi}{\lambda_{\mathrm{C}}} C_{\alpha \beta}+\frac{k_{\mathrm{B}} T}{\lambda_{\mathrm{C}} K}(1-\phi) \delta_{\alpha \beta} \\
& -\frac{1}{2} \sqrt{\frac{\Gamma}{G}} \frac{(1+p)^{2} \phi \theta}{\sqrt{\lambda_{\mathrm{C}} \lambda_{\mathrm{S}}}} \\
& {\left[\left(I_{1}^{\mathrm{S}} \delta_{\gamma \in}-S_{\gamma \in}\right)\left(C_{\alpha \gamma} S_{\beta \in}+C_{\beta \gamma} S_{\alpha \in}\right)-\frac{4}{3} I_{2}^{\mathrm{S}} C_{\alpha \beta}\right], }
\end{aligned}
$$

$$
\begin{aligned}
\left.\frac{\partial S_{\alpha \beta}}{\partial t}\right|_{\text {diss }}= & -\frac{(1+p)^{2} p \phi}{\lambda_{\mathrm{S}}}\left(\frac{1}{3} I_{1}^{\mathrm{S}} S_{\alpha \beta}-S_{\alpha \gamma} S_{\gamma \beta}\right)+\frac{p \phi}{\lambda_{\mathrm{S}}}\left(S_{\alpha \beta}-\frac{3 \delta_{\alpha \beta}}{I_{2}^{\mathrm{S}}}\right) \\
& -\frac{K}{2 k_{\mathrm{B}} T} \sqrt{\frac{\mathrm{G}}{\Gamma}} \frac{(1+p)^{2}(1-\phi) \theta}{\sqrt{\lambda_{\mathrm{C}} \lambda_{\mathrm{S}}}} \\
& \times\left(C_{\alpha \gamma} S_{\beta \gamma}+C_{\beta \gamma} S_{\alpha \gamma}-\frac{2}{3} I_{1}^{\mathrm{C}} S_{\alpha \beta}\right) .
\end{aligned}
$$

Eq. (14a) is the dissipative contribution to the conformation tensor dynamics. For $\theta=0$, we obtain the relaxation terms of the UCMM and our set of equations reduces to an uncoupled multi-mode model. For $\theta \neq 0$ and $p \neq-1$, we have a non-trivial coupling of the droplet phase to the matrix. This means that the droplet deformation induces changes in the average conformation of the molecules in the matrix phase. Equation (14b) represents the dissipative contributions to the droplet relaxation dynamics. The first term on the right-hand side of Eq. (14b) accounts for oblate droplets and the second term accounts for prolate droplets. The third term captures the influence of the local polymer conformation in the matrix phase on the shape and relaxational behavior of the droplets, and it gives oblate droplets for $\theta>0$.

For $p=-1$, i.e., equi-viscosity blends, the oblate term in the droplet configuration equation and the coupling terms drop out and we recover a model of the MM type together with a UCMM. Note that for equi-viscosity blends, the droplet deformation in the MM model is described by the upper-convected time derivative and the prolate relaxation term since the degree of non-affine motion was taken as $f_{2}=5 /(3-2 p)$ in Maffettone and Minale (1998); consequently, it vanishes for equi-viscosity liquids. For $p \neq-1$, the prolate contribution in the droplet configuration equation and the coupling terms become different from zero, and the droplets deform into non-axisymmetric shapes for start-up of steady shearing flow. In the MM model this effect is obtained through the inclusion of the Gordon-Schowalter derivative into the droplet configuration equation. For $p \rightarrow 0^{-}$(i.e., $\mathrm{Ca} \rightarrow \infty$, cf. the next section), the interfacial tension between the two phases is negligible, and variations of the droplet shape are due to the coupling of the viscoelastic matrix to the droplets, i.e., the last term in Eq. (14b). Vice versa, droplet deformation induces structural changes in the matrix fluid via the coupling term in Eq. (14a). The coupling term in Eq. (14a) is a consequence of the modeling approach and it ensures thermodynamic consistency of the partial differential equations. Consequently, droplet deformation is always coupled with structural changes in the matrix for thermodynamic consistency requirements. However, for specific choices of the physical parameters $\left(G, \lambda_{\mathrm{C}}\right)$ and $\left(\Gamma, \lambda_{\mathrm{S}}\right)$, the coupling terms in Eq. (14a) can be small compared to those in Eq. (14b) so that there is only a small effect of droplet deformation on the structural properties of the matrix. Note that in the limit $p \rightarrow 0^{-}$, the degree of non-affine motion as defined in Maffettone and Minale (1998) is $f_{2}=5 / 3>1$. For this value of $f_{2}$, the deformation of the droplets is not described by the usual Gordon-Schowalter derivative since $f_{2} \notin[-1,1]$. For $p \rightarrow-\infty$ (i.e., $\mathrm{Ca}=0$ ), the droplet relaxation terms in Eq. (14a) become the dominant ones, and 
the drops behave like rigid particles (note that the coupling term in Eq. (14b) vanishes for $\mathbf{S}=\delta$, i.e., in the undeformed state or for non-deformable droplets). For the dilute regime, $\phi \rightarrow 0$, only the coupling term in Eq. (14b) is different from zero, which predicts oblate droplet shapes. However, the presence of the droplet phase allows changes in the local polymer conformation of the matrix phase constituents.

The elastic extra stress tensor of the blend is the linear combination of the extra stress tensor of the two components. With Eq. (8), the general expression of Eq. (5) for the extra stress tensor is equivalent to

$$
\begin{aligned}
\sigma_{\alpha \beta}= & \sigma_{\alpha \beta}^{\mathrm{C}}+\sigma_{\alpha \beta}^{\mathrm{S}}=n_{C} K(1-\phi) C_{\alpha \beta}-n_{C} k_{B} T(1-\phi) \delta_{\alpha \beta} \\
& +\Gamma \phi\left(I_{1}^{S} S_{\alpha \beta}-S_{\alpha \gamma} S_{\gamma \beta}-\frac{2}{3} I_{2}^{S} \delta_{\alpha \beta}\right) .
\end{aligned}
$$

For the dilute regime $(\phi \rightarrow 0)$, we have mainly a stress contribution due to the matrix phase and the droplet configuration is governed by the coupling term in Eq. (14b). For the concentrated regime $(\phi \rightarrow 1)$, the extra stress is due to the droplet phase and the nonlinear coupling terms are the only contributions in the conformation tensor evolution equation, Eq. (14a). Equation (15) is consistent with the extra stress tensor obtained by Grmela et al. (2001).

\section{Sample calculations for various types of homogeneous deformations}

The set of continuum equations (Eqs. 4a, 4b, 4c), derived in the previous section, has been solved for various combinations of physical parameter values to determine its characteristic features for various homogeneous flows, characterized by the velocity gradient tensor, $\nabla \mathrm{v}$. These equations have been rendered dimensionless using quantities $\bar{t}=t / \sqrt{\lambda_{\mathrm{C}} \lambda_{\mathrm{S}}}, \tilde{C}=K / k_{\mathrm{B}} T C, \tilde{S}=S$, and $\tilde{\sigma}=$ $\sigma /(\sqrt{G \Gamma})$. In all that follows, we omit the tilde over dimensionless quantities. The equations have been solved for various ratios of elastic strengths $(G / \Gamma)$ and relaxation times $\left(\lambda_{\mathrm{C}} / \lambda_{\mathrm{S}}\right)$, i.e., viscosity ratios, $p=-\left(\Gamma \lambda_{\mathrm{S}}\right) /$ $\left(G \lambda_{\mathrm{C}}\right)$, various droplet concentration regimes, $\phi$, and coupling parameter values, $\theta$, using a fourth-order Runge-Kutta scheme. The Capillary Number is defined as $\mathrm{Ca}=\eta_{\mathrm{c}} R /\left(\Gamma^{\prime} \lambda_{\mathrm{S}}\right)$ in analogy with Edwards and Dressler (2003), i.e., the intrinsic time scale of the viscoelastic interface is adopted in the definition of the Capillary Number instead of the time scale related to the velocity gradient.

The groups that we adopt to render the system of equations dimensionless thus only involve characteristic material properties and intrinsic time scales. We feel that once one incorporates matrix-phase viscoelasticity into the problem, this is a more physically realistic definition; however, this is largely a matter of taste and a ripe subject for future debate. However, using the standard definition of $\mathrm{Ca}$, this quantity depends on space and time for transient and non-homogeneous flow fields; hence the advantage gained in using a $\mathrm{Ca}$ defined in terms of material properties only.

With the above relationship, we see that the Capillary Number is fixed once the viscoelastic properties of the two phases have been specified: $\mathrm{Ca}=-1 / p$. The reason for the Capillary Number being the only dimensionless group lies in the choice of a single elastic modules, $\Gamma$, and the corresponding relaxation time, $\lambda_{\mathrm{S}}$, to define the physical properties of the droplet phase, i.e., we did not introduce an additional droplet viscosity, $\eta^{*}$, but set $\eta_{d}=\Gamma \lambda_{S}$ in analogy with the UCMM.

In the following, we discuss the basic features of the set of continuum equations derived in the previous section. We will focus mainly on (i) a dilute model blend $(\mathrm{Ca}=4)$ whose linear viscoelastic properties are defined as $G / \Gamma=4$ for the ratio of elastic strengths and $\lambda_{\mathrm{C}}$ $\lambda_{\mathrm{S}}=1$ for the ratio of relaxation times, and (ii) a model blend $(\mathrm{Ca}=2)$ also with $\lambda_{\mathrm{C}} / \lambda_{\mathrm{S}}=1$. Furthermore, the equations are also solved for $\mathrm{Ca}=0.1,1$, and 10 in order to discuss the predictions over a larger range of Capillary Numbers, and to compare our equations with the predictions of the MM Model and the ALS Model to examine the contribution of the viscoelastic matrix phase. The dispersed phase concentration is taken as $\phi$ $=0.1$ in most sample calculations. The morphological and viscometric properties of the model blend will be studied for various coupling parameter values. In the MM Model, the Gordon-Schowalter derivative has been adopted to describe droplet deformation in blends of Newtonian liquids with a viscosity ratio different from unity. In this article, we do not want to adopt the Gordon-Schowalter derivative since it is an irreversible contribution to the system dynamics which does not lead to dissipation of mechanical energy; i.e., it does not contribute to Eq. (12), even though it is irreversible! Instead, we wish to study the effect of the oblate relaxation term in Eq. (14b) and of the coupling terms in Eqs. (14a and 14b) on the rheological and microstructural properties of the blend. Finally, we will fit our model to experimental data. For this purpose the viscosity ratio will be used as an independent quantity and the EWM Model will be adopted to account for the shear thinning behavior of the matrix fluid.

\section{Simple shear flow}

First, we investigate the system equations for start-up of steady shear flow, i.e., $\nabla_{2} v_{1}=\dot{\gamma}$, focusing on the nonlinear rheological features of the two coupled modes description of the blend. Figure 1 illustrates the transient behavior of the scalar invariants of the structural 
variables as a function of shear strain, $\gamma$, for a given shear rate, $\dot{\gamma}$, and two values of the coupling parameter, $\theta$, according to Eqs. (4a), (4b), (4c), (6a), (6b),(14a)and (14b). In this case, all fields are spatially homogeneous and the integrals in Eq. (13) give the total system volume, $\Omega$. The calculations are for the $\mathrm{Ca}=4$ model blend, i.e., $p=-0.25<0$ to satisfy thermodynamic admissibility criteria for the system equations. We note that the invariants of $\mathbf{C}$ (Fig. 1a-c) increase upon start-up of steady shear flow, and that they each attain a value greater than their equilibrium values. The solid lines in Fig. 1a-c correspond to the single-mode UCMM, and they can be obtained analytically solving this linear viscoelastic model. The first and the second invariant of $\mathbf{S}$ (Fig. 1d,e) show a strongly non-linear behavior which is related to the increase of the surface area of the ellipsoidal inclusions. The third invariant of $\mathbf{S}$ is always unity, as prescribed by the constant-volume constraint. Figure $1 \mathrm{~g}$ displays the total rate of mechanical energy dissipation, $\left[H_{\mathrm{m}}, H_{\mathrm{m}}\right] / \Omega$, according to Eq. (13).

Figure 2 shows the transient behavior of the conformation tensor, $\mathbf{C}$, which describes the average conformation and orientation of the molecules in the matrix phase. We notice that the 11- and the 12-components of the conformation tensor attain a steady state before approximately one shear strain unit has been applied, whereas the 22- and the 33-components reach their steady-state values only at much higher strain values. The non-trivial behavior of the latter components is directly attributable to the fact that $\theta \neq 0$ in Fig. $4 \mathrm{~b}$. Only for $\theta=0$ does the conformation tensor equation
(Eq. 4b), reduce to the UCMM, for which $C_{22}=C_{33}=1$ in simple shear flow.

The morphological properties of the polymer blend are represented by the average magnitude of the three semiaxes of the ellipsoidal droplets and the average orientation of the droplets with respect to the flow direction. The semiaxes of the droplets are the square roots of the eigenvalues of the droplet configuration tensor, $\mathbf{S}$. The droplet semiaxes in the flow direction, in the direction of the shear gradient, and in the direction of the vorticity axis are denoted with $L, B, W$, respectively. Upon inception of weak steady shear flow, $\dot{\gamma}<1$, the major droplet axis, $L$, increases and the minor droplet axis, $B$, decreases. However, the vorticity axis, $W$, can be greater than, smaller than, or equal to unity upon start-up of flow and the droplets are thus either oblate or prolate: one has oblate droplets for $W>1$ and prolate droplets for $W<1$. The orientation angle, $\chi$, is the angle between the eigenvector corresponding to the largest eigenvalue of the $\mathbf{S}$-tensor and the flow direction and it is defined as $\chi=1 / 2 \arctan \left[2 S_{12} /\left(S_{11}-S_{22}\right)\right]$.

Figure 3 displays the start-up behavior of the three droplet semiaxes upon inception of steady shear flow for a fixed value of the coupling parameter and three different shear rates (Fig. 3a), as well as for a fixed shear rate and three coupling parameter values (Fig. 3b). We see that the model predicts a transition from the prolate to oblate droplet configurations for increasing shear rate or increasing coupling parameter value when $\theta \neq 0$. At intermediate shear rates and coupling parameter values, there is a competition between the prolate and the oblate
Fig. 2 The components of the conformation tensor, $\mathbf{C}$, for start-up of steady shear flow for three shear rates, $\dot{\gamma}=0.05$ (solid lines), 0.1 (dashed lines), and 0.2 (dotted lines) as a function of shear strain, $\gamma$, for the $\mathrm{Ca}=4$ blend with dispersed phase concentration $\phi=0.1$. The phenomenological coupling parameter is $\theta=0.1$

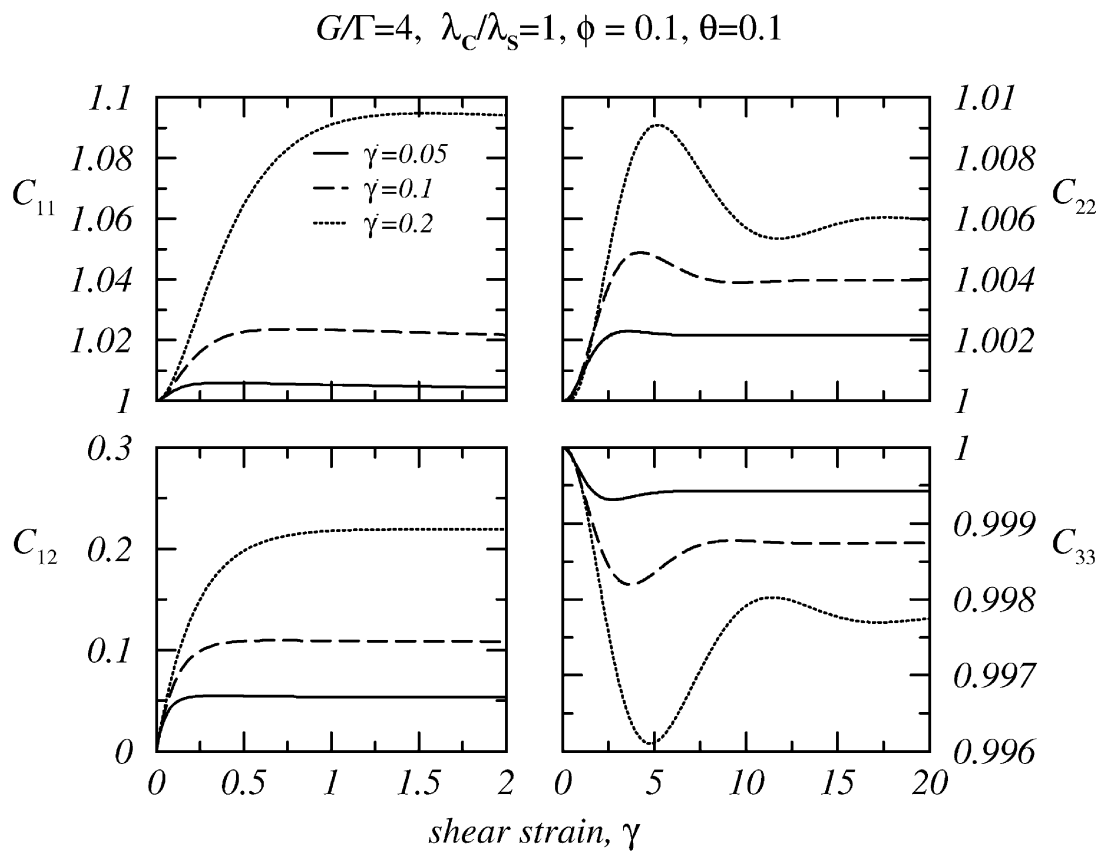


Fig. 3 The same as Fig. 2 for the average semiaxes of the ellipsoidal droplets, $L, B, W$, and the orientation angle, $\chi$, for start-up of steady shear flow for (a) constant coupling parameter, $\theta=0.1$, and three shear rates $\dot{\gamma}=0.05$ (solid lines), 0.1 (dashes lines), 0.2 (dotted lines); (b) constant shear rate, $\dot{\gamma}=0.05$ and three coupling parameter values $\theta=0.1$ (solid lines), 0.2 (dashes lines), and 0.4. (dotted lines). Depending on the shear rate and the magnitude of the coupling parameter, the model predicts either oblate droplets $(W>1)$ or prolate droplets $(W<1)$. For $\dot{\gamma}=0.1$ and small shear strains, we observe a competition between oblate and the prolate configurations a)

$$
G / \Gamma=4, \lambda_{\mathrm{C}} / \lambda_{\mathrm{s}}=1, \phi=0.1, \theta=0.1
$$

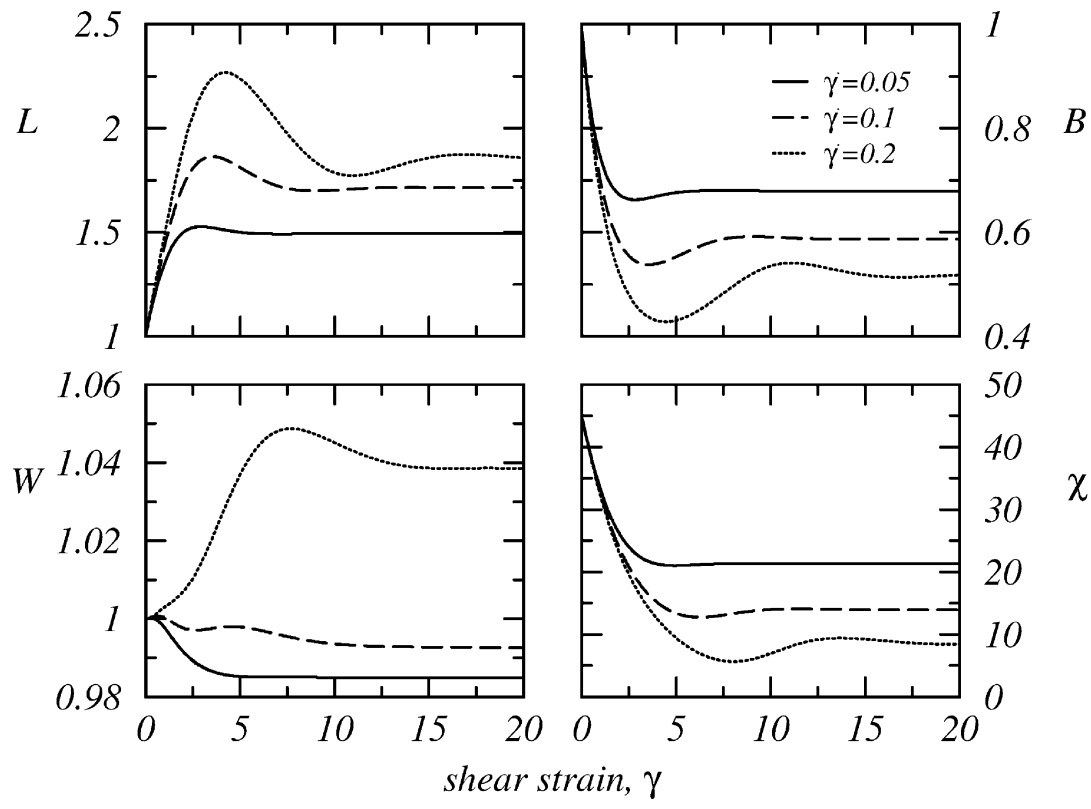

b)

$$
G / \Gamma=4, \lambda_{\mathrm{C}} / \lambda_{\mathrm{s}}=1, \phi=0.1, \gamma=0.05
$$
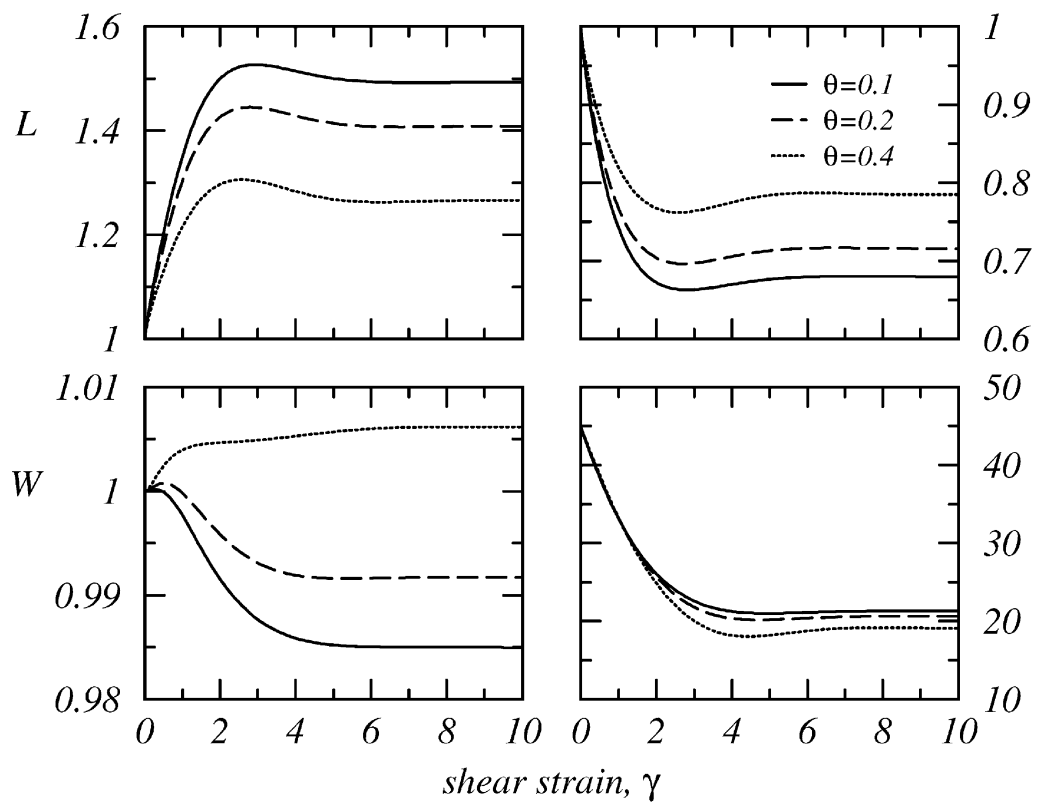

$\chi$ droplet deformations that ends up in a prolate nonequilibrium state for high shear strains.

Another example of the competition between oblate and prolate droplet shapes is illustrated in Fig. 4 for the $\mathrm{Ca}=2$ blend with dispersed phase concentration $\phi=0.1$. In Fig. 4, the vorticity axis for an ellipsoidal droplet is plotted as a function of shear strain for start-up of steady shearing flow, $\dot{\gamma}$, for five coupling parameter values. It is seen that the droplets deform into prolate ellipsoids at high shear strains. The inset in Fig. 4 shows the vorticity axis for small shear strains immediately after start-up of flow. We see that for higher values of the coupling parameter, $\theta$, the droplets deform into oblates immediately after start-up of steady shearing flow. At a critical shear strain, the vorticity axis reaches a maximum and then decreases below the equilibrium value, where the prolate steady state is achieved at high shear strains. Levitt et al. (1996) report an increase of the 


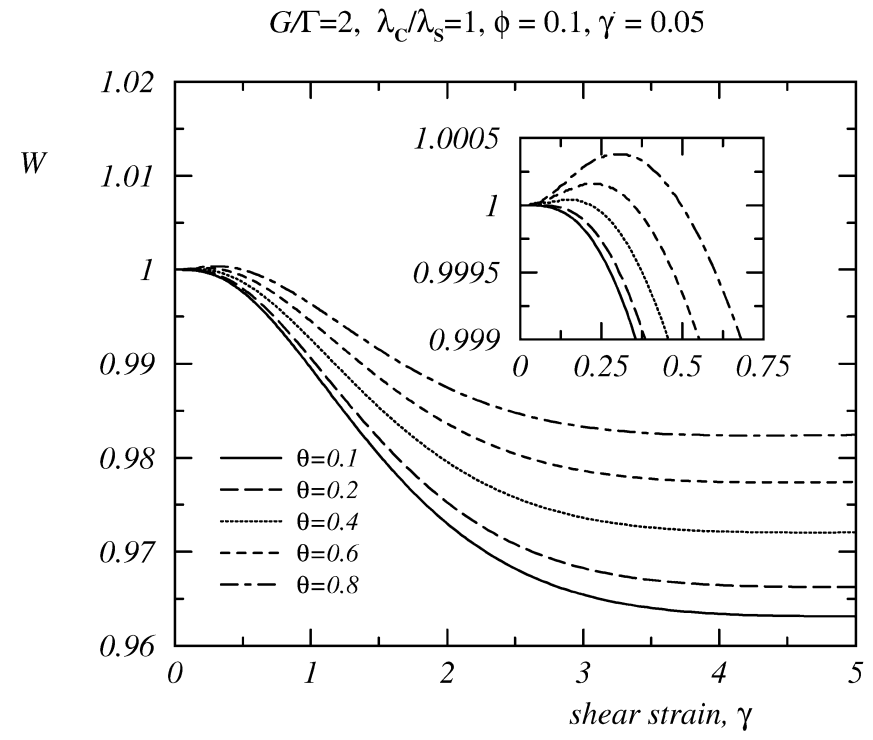

Fig. 4 The vorticity axis of ellipsoidal droplets for a $\mathrm{Ca}=2$ blend with dispersed phase concentration $\phi=0.1$, five coupling parameter values, $\theta=0.1$ (solid line), 0.2 (long-dashed), 0.4 (dotted), 0.6 (dashed), 0.8 (dot-dashed), and shear rate $\dot{\gamma}=0.05$. The inset shows the behavior of the vorticity axis immediately after start-up of steady shear flow. The vorticity axis reaches a maximum at a critical shear strain and evolves into a prolate steady state

vorticity axis upon start-up of steady shearing flow for a single polypropylene droplet in a viscoelastic polystyrene matrix with $G / G_{\mathrm{d}} \approx 3$ and in other droplet/matrix pairs of synthetic polymers with $1<G / G_{\mathrm{d}}<9$, where $G_{\mathrm{d}}$ is the elastic modulus of the dispersed phase. In these experi- ments, a single droplet was subjected to simple shear flow in a parallel-plate geometry while the droplet was observed with a camera. A maximum value of 1.4 was observed for the vorticity axis, $W$, relative to the equilibrium droplet radius in the $G / G_{\mathrm{d}} \approx 3$ blend. Levitt et al. (1996) attributed this droplet behavior to matrix phase viscoelasticity and they used a phenomenological force balance at the critical shear strain to capture the effect of matrix viscoelasticity on droplet deformation.

In Fig. 5 we display the transient viscometric properties of the $\mathrm{Ca}=4$ blend for three shear rates. The continuum equations derived in the previous section predict a typical non-linear viscoelastic response as encountered often in synthetic polymer rheology. The right column in Fig. 5 displays the shear stress, $\sigma_{21}$, and the shear viscosity, $\eta_{\mathrm{S}}=\sigma_{21} / \dot{\gamma}$, as a function of shear strain. The shear stress shows a strongly non-linear transient behavior with over- and undershoots, which increase with increasing shear rate. The first normal stress difference, $N_{1}=\sigma_{11}-\sigma_{22}$, is positive. Similarly to the shear stress, it shows a non-linear behavior with over- and undershoots, and it is of the same order of magnitude as the shear stress. The second normal stress difference, $N_{2}=\sigma_{22}-\sigma_{33}$, is negative and approximately half the magnitude of the first normal stress difference. The normal stress differences attain a steady state at higher shear strains compared to the shear stress. Finally, the transient shear viscosity, $\eta_{\mathrm{S}}$, is also reported in Fig. 5; it displays the characteristic shear-thinning behavior. This is illustrated more clearly in Figs. 6a and $7 \mathrm{a}$ where we present the steady-state viscometric properties for the $\mathrm{Ca}=4$ and the $\mathrm{Ca}=2$ blend.
Fig. 5 The same as Fig. 2 for the transient viscometric properties of the $\mathrm{Ca}=4$ blend. The left column shows the start-up behavior of the shear stress and the shear viscosity, $\sigma_{12}$ and $\eta_{\mathrm{S}}$, while the right column displays the start-up behavior of the normal stress differences, $N_{1}$ and $N_{2}$

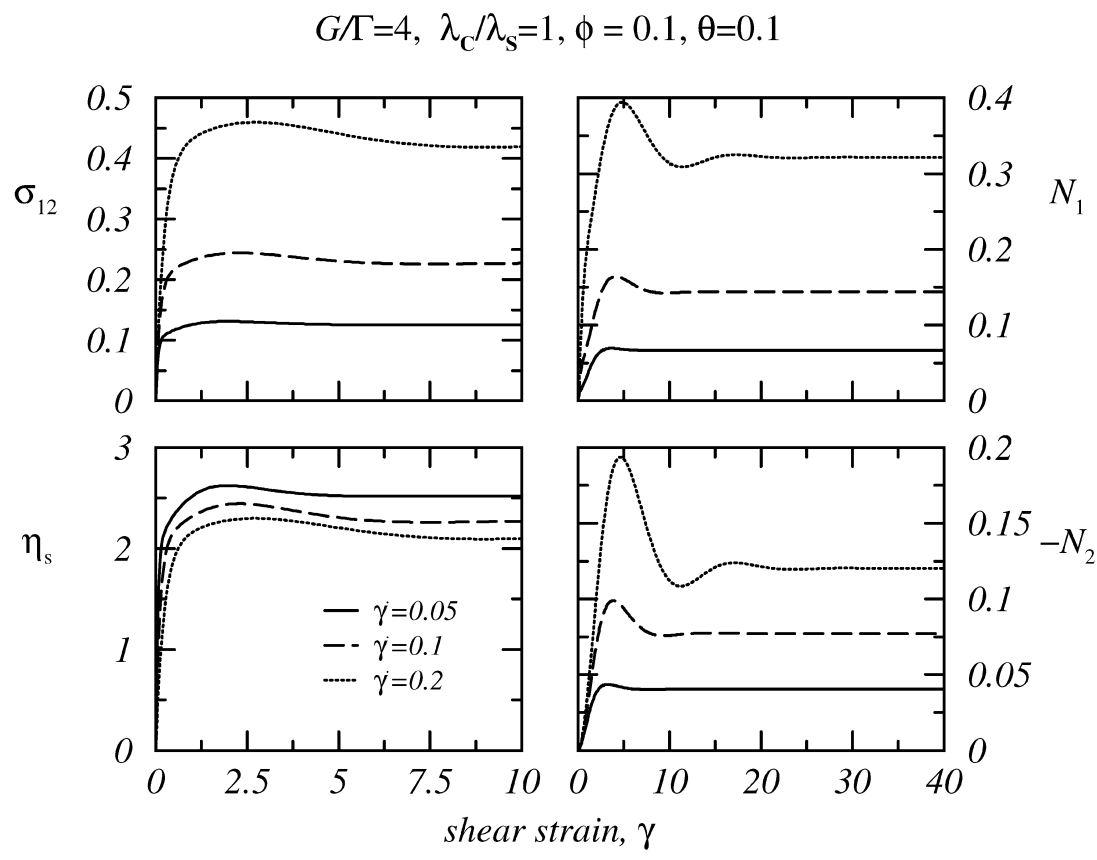


Fig. 6 The (a) steady-state viscometric; (b) morphological properties of the $\mathrm{Ca}=4$ model blend with dispersed phase concentration, $\phi=0.1$, for three coupling parameter values: $\theta=0.05$ (solid lines), 0.1 (dashed lines), and 0.15 (dotted lines) in simple shear flow: (a) shows the steady state viscometric functions $\eta_{\mathrm{S}}, \Psi_{1}, \Psi_{2}$ and the ratio $\Psi_{1} / \Psi_{2}$; (b) displays the droplet semiaxes $L, B, W$, and the orientation angle, $\chi$ a) $\quad G / \Gamma=4, \lambda_{\mathrm{c}} / \lambda_{\mathrm{s}}=1, \phi=0.1$

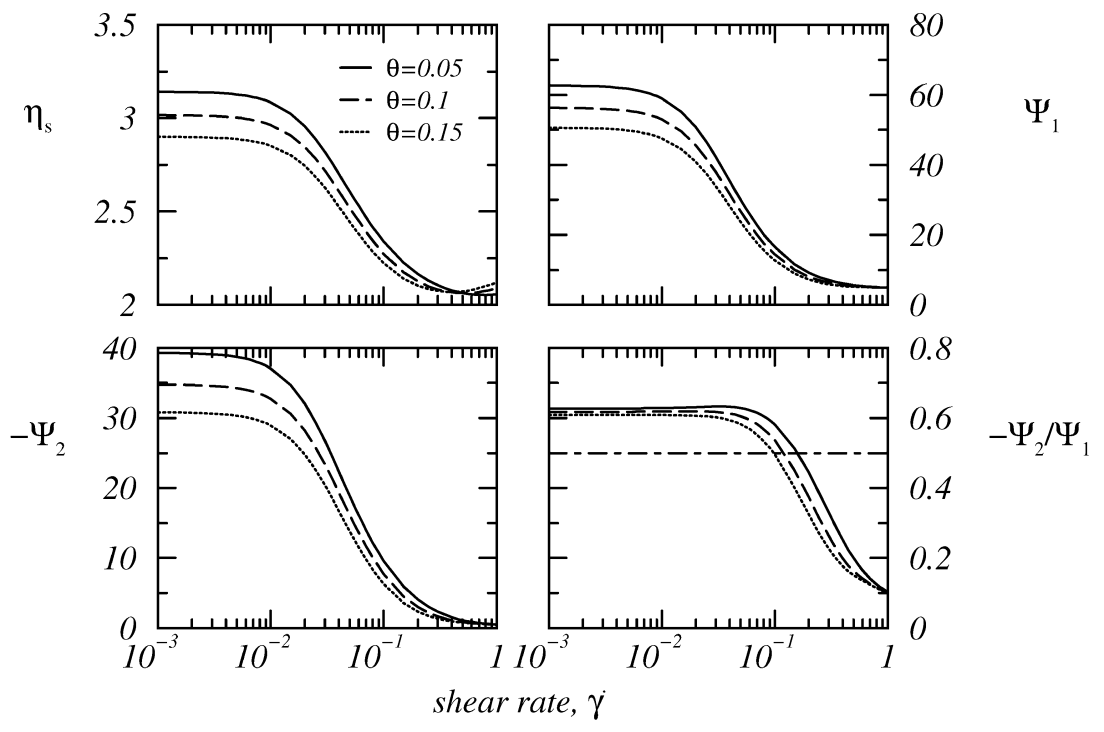

b) $\quad G / \Gamma=4, \lambda_{\mathrm{C}} / \lambda_{\mathrm{s}}=1, \phi=0.1$

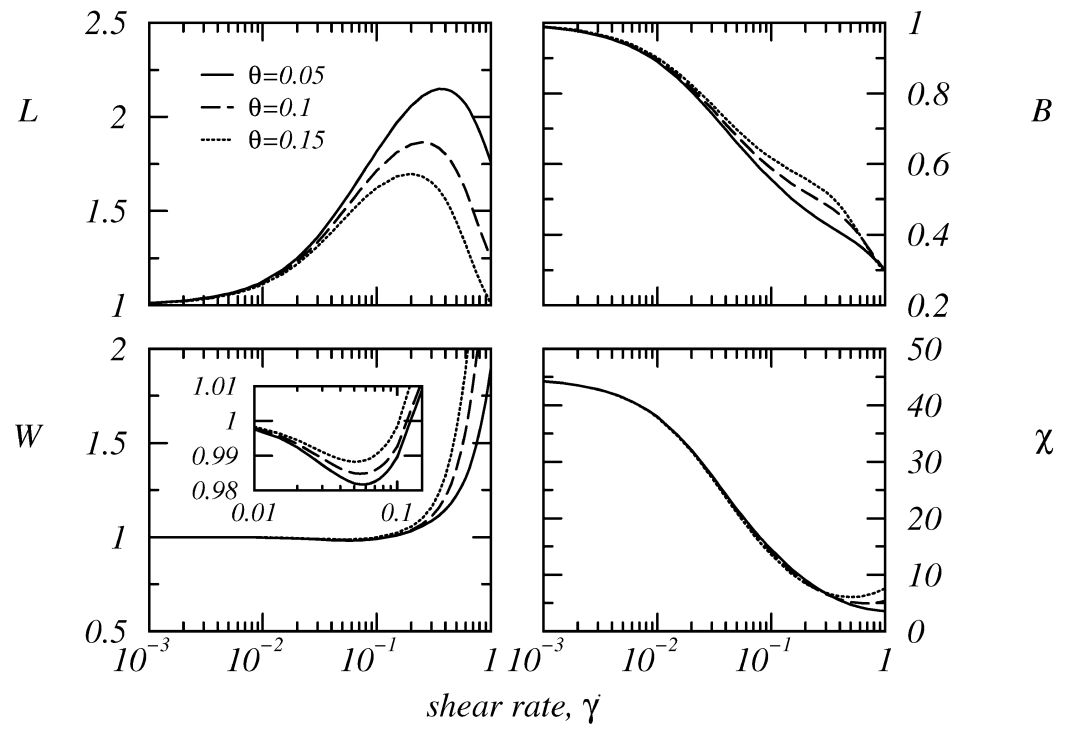

It is interesting to determine correlations between the magnitude of the semiaxes of the ellipsoidal droplets and the viscometric functions of the material. Such a procedure is discussed, e.g., in Yu et al. (2002a), who propose correlations between the viscometric properties of the blend and higher-order Taylor deformation parameters of the type, $D_{X Y}=\left(X^{2}-Y^{2}\right) /\left(X^{2}+Y^{2}\right)$, where $X, Y=L, B, W$ denote the semiaxes of the drop. Possibly, it is more instructive to establish correlations between the semiaxes and the viscometric functions instead of defining generalized Taylor deformation parameters. A comparison of Figs. 5 and 3a shows, e.g., that the first normal stress difference correlates with the major semi- axis, $L$, and the second normal stress difference correlates with the minor droplet semiaxis, $B$. However, such correlations should be used with caution due to the nonlinear character of the underlying dynamics. We will comment further on such correlations below.

In Figs. 6 and 7 we present the steady-state viscometric and morphological properties of the $\mathrm{Ca}=4$ and the $\mathrm{Ca}=2$ model blends in shear flow. Figs. $6 \mathrm{a}$ and $7 \mathrm{a}$ show the viscometric functions for the two model blends with dispersed phase concentration $\phi=0.1$. We observe a shear-thinning behavior in the three viscometric functions. The plateau values of the viscometric functions decrease as the coupling parameter is 
Fig. 7 The same as Fig. 6 for a $\mathrm{Ca}=2$ model blend with dispersed phase concentration $\phi$ $=0.1$ and three coupling parameter values: $\phi=0.1$ (solid lines), 0.2 (dashed lines), and 0.4 (dotted lines). The vorticity axis of the droplets undergoes a transition form a prolate to an oblate configuration regime with increasing shear rate a)

$$
G / \Gamma=2, \lambda_{c} / \lambda_{s}=1, \phi=0.1
$$

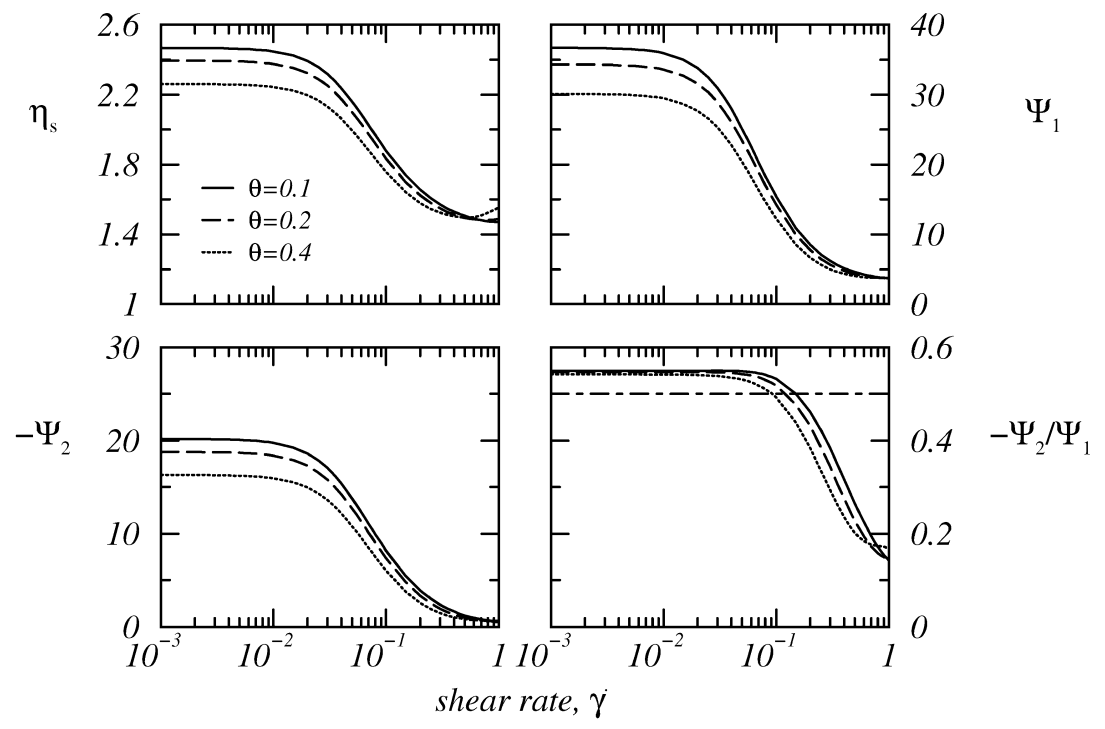

b)

$$
G / \Gamma=2, \lambda_{\mathrm{C}} / \lambda_{\mathrm{s}}=1, \phi=0.1
$$

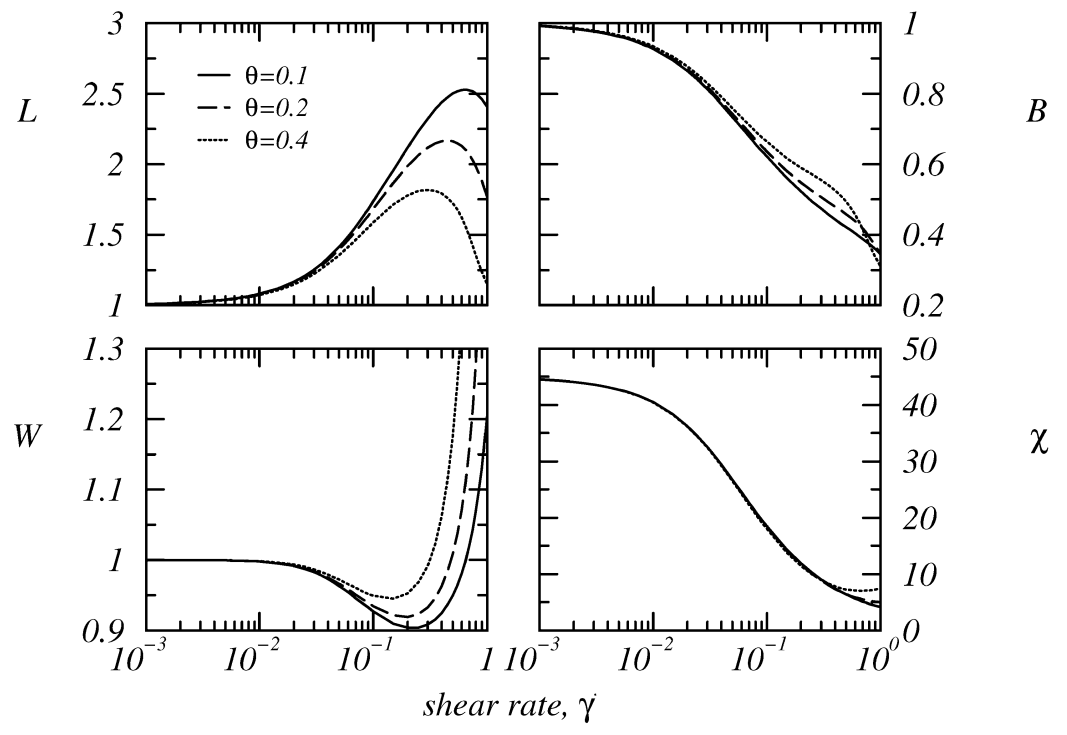

increased. The power-law index of the shear viscosity curve in the shear-thinning region decreases with increasing coupling parameter. However, the power-law indices of the steady-state normal stress coefficients increase with increasing coupling parameter. This is seen more clearly in Fig. 8, where all viscometric functions have been normalized with respect to the zero shear-rate values of the respective flow curves. For both model blends $(\mathrm{Ca}=4$ and 2), the shear-thinning regime sets in at shear rates of $\dot{\gamma} \approx 0.01$, and there is a broad transition from the plateau in the low shear-rate regime to the power-law region in the intermediate and high shear-rate regimes. For $\dot{\gamma} \approx 0.2$, the steady-state viscometric functions start to deviate from power-law behavior and a second Newtonian plateau is reached. For even higher shear rates, the onset of a shearthickening region is observed where the shear viscosity begins to increase with increasing shear rate. A dimensionless value of $\dot{\gamma} \approx 1$ corresponds to extremely high shear rates in reality since we have already left the power-law region of the flow curves. Finally, the ratio $-\Psi_{2} / \Psi_{1}$ is reported in Figs. $6 \mathrm{a}$ and $7 \mathrm{a}$ which varies between 0.1 and 0.6 for both blends. The dot-dashed lines in Figs. $6 \mathrm{a}$ and $7 \mathrm{a}$ are the predictions of the MM Model, $\Psi_{2} / \Psi_{1}=-0.5$. We realize that our set of equations allows for a more general behavior of the ratio of normal stress differences compared to the MM Model. 
Fig. 8 The same as Fig. 6a for the steady-state viscometric functions, normalized with respect to their zero shear-rate values

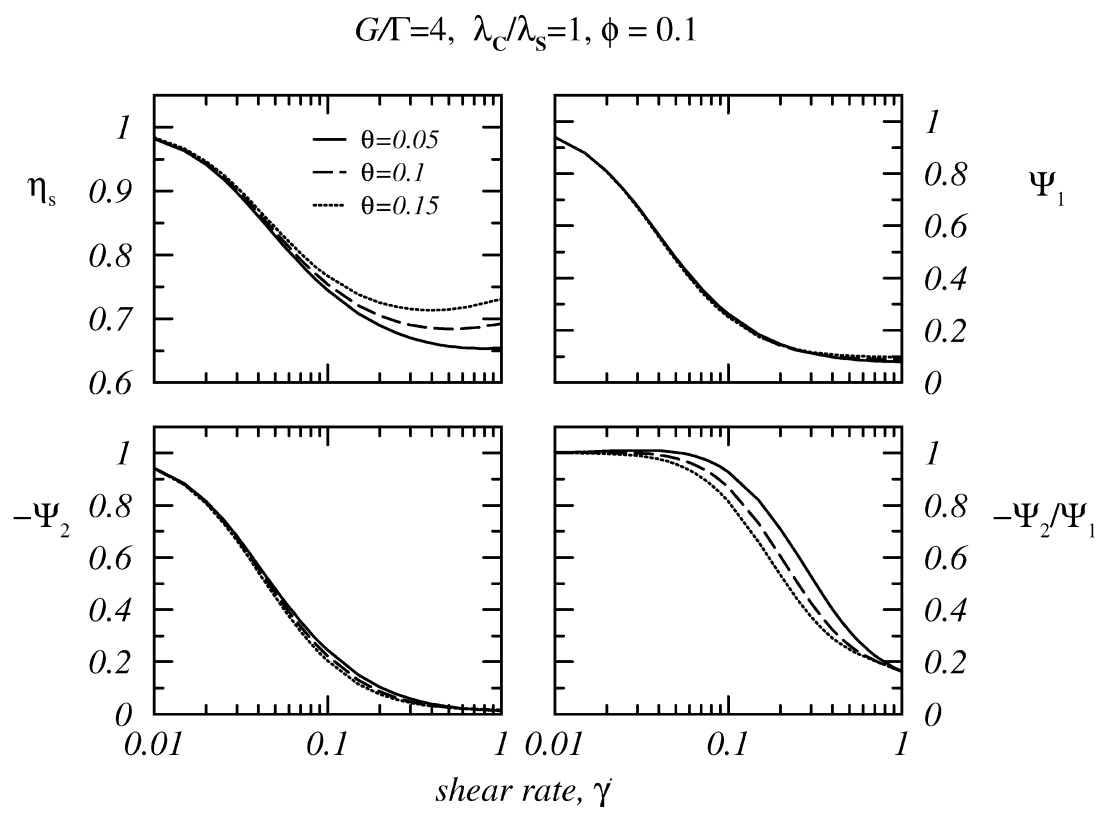

Note that our set of continuum equations $(4 a, 4 b, 4 c)$, allows us to compute steady-state viscometric properties over an extremely large shear rate regime that extends from a Newtonian plateau to a power-law regime at low and intermediate shear rates, to a second Newtonian plateau with perhaps the onset of a shear-thickening region at extremely high shear rates. The prediction of steady-state viscometric blend properties is critical for both the ALS Model and the MM Model. As has been pointed out, e.g., in Jansseune et al. (2001), the original ALS Model does not yield a steady-state shear stress for start-up of simple shear flow. The problem with the MM Model, Eq. (1), is that it yields a critical shear rate, $\dot{\gamma}_{\mathrm{C}}=f_{1} /\left(\sqrt{f_{2}^{2}-1} \lambda\right)$. Beyond $\dot{\gamma}_{\mathrm{c}}$, the droplet shape tensor (and hence the extra stress tensor) increases to infinity for start-up of steady shear flow. Therefore, the steady-state shear viscosity and the first normal stress difference can be predicted only over a limited range of shear rates for the MM Model-see Yu et al. (2002a) (Fig. 5). The present approach does not make use of the mixed convected derivative for the droplet configuration tensor, but is expressed in terms of the upper-convected derivative, since $\mathbf{S}$ is a contravariant, second-rank tensor. However, this corresponds to $f_{2}=1$ which means $\dot{\gamma}_{\mathrm{c}} \rightarrow \infty$ for the uncoupled set of evolution equations in simple shear flow.

Figures $6 \mathrm{~b}$ and $7 \mathrm{~b}$ show the steady-state morphological properties of the ellipsoidal inclusions in the viscoelastic matrix. For both blends, we observe a strong increase in the major droplet axis, $L$, which reaches a maximum at $\dot{\gamma} \approx 0.4$ and $\dot{\gamma} \approx 0.6$ for the $\mathrm{Ca}=4$ and the $\mathrm{Ca}=2$ model blends, respectively. This increase in $L$ correlates (i) with flow alignment of the droplet inclusions since the orientation angle, $\chi$, decreases with increasing shear rate, and (ii) with a strong increase of the vorticity axis, $W$. The minor droplet axis, $B$, is a decreasing function of shear rate over the entire range of shear rates investigated herein. A comparison with Figs. 6a and 7a shows that the increase in the major droplet axis, $L$, correlates with the beginning of the power-law region that is observed in the steady-state viscometric functions. The decrease of the major droplet axis, $L$, and the strong increase of the vorticity axis, $W$, correlate with the approach of the viscometric functions to a second Newtonian plateau. Also, the orientation angle approaches a plateau value for high shear rates, and for the $\mathrm{Ca}=4$ blend an increase of $\chi$ is observed for $\theta=0.15$ at very high shear rates.

Summarizing the results of Figs. $6 \mathrm{~b}$ and $7 \mathrm{~b}$, we can identify several steady-state droplet shape regimes. For small shear rates, $10^{-3}<\dot{\gamma}<10^{-2}$, the steady-state droplet shape lies in the prolate deformation regime. For the $\mathrm{Ca}=4$ and the $\mathrm{Ca}=2$ blends, the major droplet axis increases with increasing shear rate and the minor droplet axis decreases, whereas the vorticity axis, $W$, remains almost unity. The droplets are non-symmetric, $\mathrm{B} \neq \mathrm{W}$. In the intermediate shear rate regime, $10^{-2}<\dot{\gamma}<10^{-1}$, we observe a further stretching of the droplets into prolates, which manifests as a further increase of the $L$-axis and a further decrease of the $B$-axis. Simultaneously, the vorticity semiaxis decreases below unity, and the droplets are stretched into long ellipsoids (cf. the inset in Fig. 6b). In the high shear rate regime, $\dot{\gamma}>0.3$, the vorticity axis shows a strong increase which is compensated by a simultaneous decrease of the major droplet axis to unity; i.e., a compression of the droplets in flow direction. Hence, we recover flat prolates 
$(B<<1)$ with major semiaxes $L \approx 1$, which are almost aligned parallel to the shearing planes $(\chi \approx 0)$ and extend into the vorticity direction.

Figure 9 displays the steady-state droplet characteristics for the equi-viscosity blend, $\mathrm{Ca}=1$, and three different droplet concentrations with a vanishing coupling parameter. In this case, the evolution equations for the conformation tensor, $\mathbf{C}$, and the droplet shape tensor, $\mathbf{S}$, decouple. The conformation tensor and the droplet shape tensor evolve according to the UCMM and a model of the MM type, respectively. We see that the vorticity axis in the steady state decreases as a function of shear rate; i.e., the MM Model predicts only prolate droplets. In order to account for oblate contributions to droplet deformation, we included a second contribution to the relaxation matrix and the non-linear coupling terms in Eqs. (7a, 7b).

The viscometric properties of an equi-viscosity blend for start-up and steady-state shear flow are presented in Figs. 10, 11, 12, and 13, where we discuss solutions of the model for several Capillary Number regimes. Figures 10, 11, 12, and 13 present the transient (Figs. 10 and 11) and the steady-state (Figs. 12 and 13) viscometric functions, as calculated from the continuum equations for the low $(\mathrm{Ca}=0.1$, first row $)$, the intermediate $(\mathrm{Ca}=1$, second row $)$, and the high $(\mathrm{Ca}=10$, third row) Capillary Number regimes and compare the results with the MM Model.

Figure 10 displays the transient shear viscosity and the first normal stress coefficient as a function of shear strain for three shear rates in the three Capillary Number regimes. The dispersed phase concentration and the coupling parameter have been taken as $\phi=0.1$ and $\theta=0.01$, respectively. Both quantities, $\eta_{\mathrm{S}}$ and $\Psi_{1}$, show a non-linear increase towards the steady state with overand under-shoots and a shear-thinning behavior, as shown before, cf. Fig. 5. Note that the magnitudes of $\eta_{\mathrm{S}}$ and $\Psi_{1}$ increase, but that the steady state is attained at approximately the same shear strain as the Capillary Number increases. For $p=-1$, i.e., $\mathrm{Ca}=1$ (second row of Fig. 10), the nonlinear behavior of the viscometric functions is due to the nonlinearity of the droplet configuration tensor equation. Figure 11 displays the negative second normal stress coefficient, $-\Psi_{2}$, and the quantity $-\Psi_{2} / \Psi_{1}$ as functions of shear strain for the same model parameters as adopted in Fig. 10. With increasing Capillary Number, the absolute magnitude of $\Psi_{2}$ and $\Psi_{2} /$ $\Psi_{1}$ increases and an overshoot is observed in $-\Psi_{2}$, which becomes more pronounced as the Capillary Number increases. The ratio $-\Psi_{2} / \Psi_{1}$ as a function of shear strain is very small for $\mathrm{Ca}=0.1$ and it varies between 0.0 and 0.7 for the $\mathrm{Ca}=1$ and the $\mathrm{Ca}=10$ blends.

Figure 12 displays the steady-state shear viscosity and the first normal stress coefficient for the three model blends $\mathrm{Ca}=0.1,1$, and 10 . We display results for three coupling parameters and droplet concentrations in the three Capillary Number regimes. For the $\mathrm{Ca}=0.1$ blend, we note that an increase of the coupling parameter increases the power-law index of the steady-shear viscosity and the first normal stress coefficient curves. This is qualitatively different from the results reported in Figs. 6a and 8, where an increase of $\theta$ resulted in a decrease of the power-law index of the steady-state shear
Fig. 9 The steady-state droplet morphology for a $\mathrm{Ca}=1$ model blend for three concentrations, $\phi=0.1$ (solid lines), 0.5 (dashed lines), 1 (dotted lines). For equiviscosity conditions, the system equations describe prolate droplet shapes

$$
G / \Gamma=1, \lambda_{\mathrm{c}} / \lambda_{\mathrm{s}}=1, \theta=0
$$

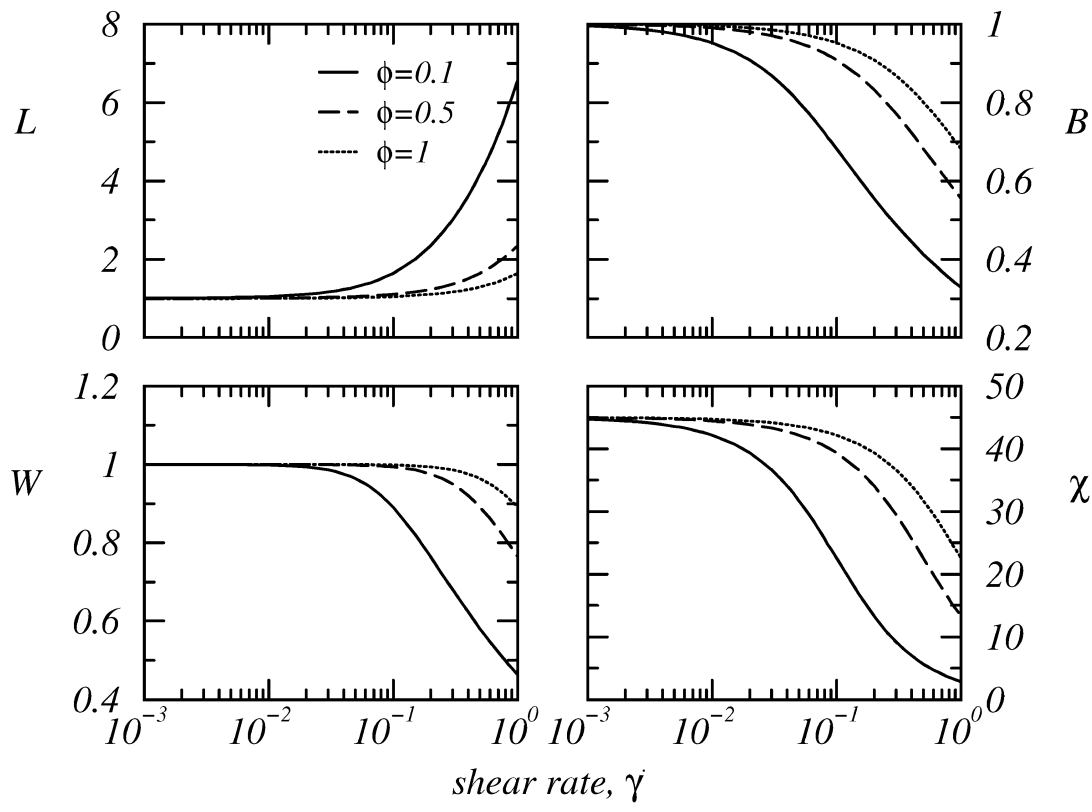


Fig. 10 The transient shear viscosity, $\eta_{\mathrm{S}}$ (left column), and the transient first normal stress coefficient, $\Psi_{1}$ (right column), for three Capillary Numbers, $\mathrm{Ca}=0.1$ (first row), 1 (second row), 10 (third row) and three different shear rates denoted in the legends within each row. The droplet concentration and the coupling parameter have been taken as $\phi=0.1$ and $\theta=0.01$, respectively
$G / \Gamma=0.1,1,10, \lambda_{\mathrm{C}} / \lambda_{\mathrm{s}}=1, \phi=0.1, \theta=0.01$
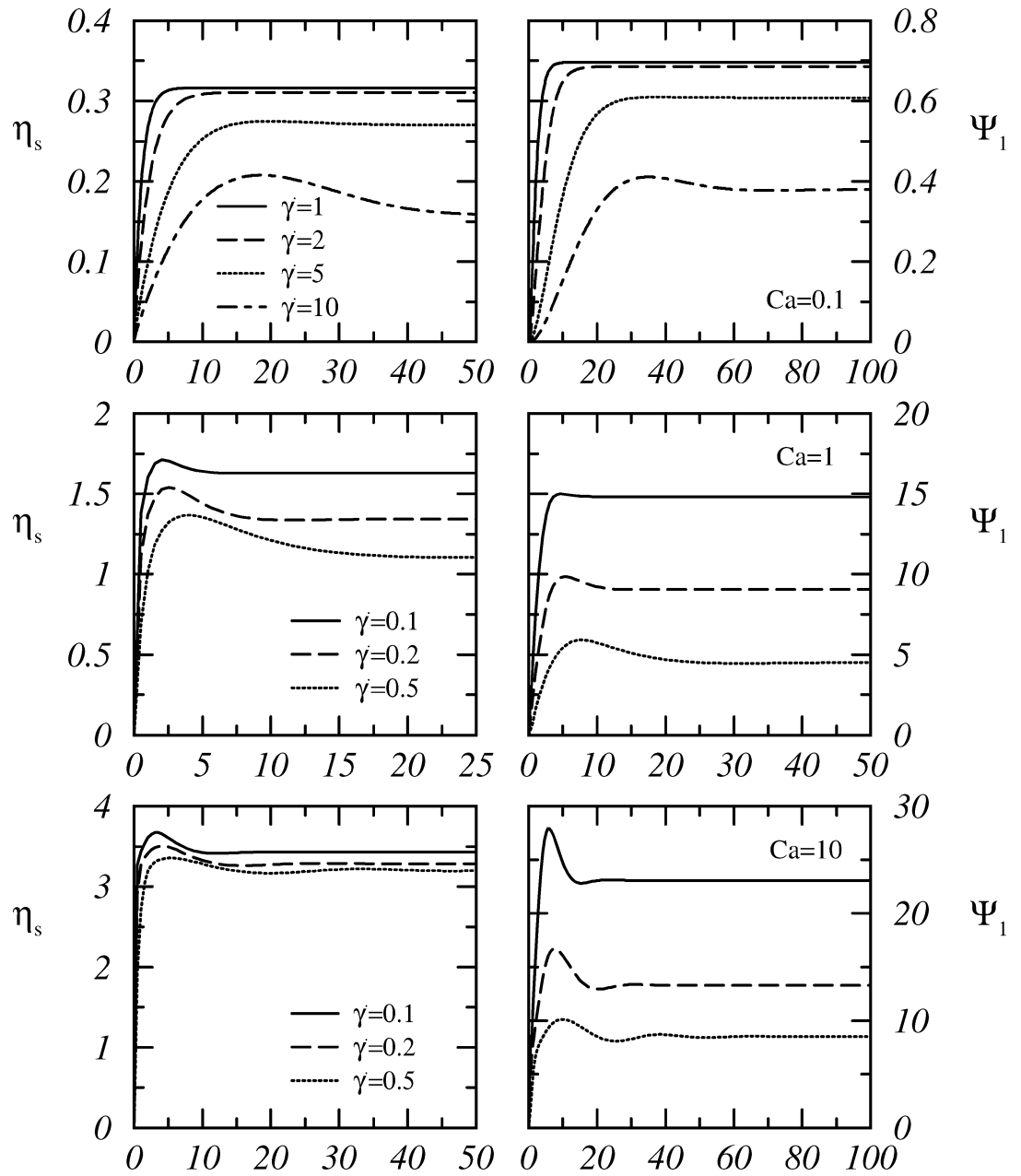

10

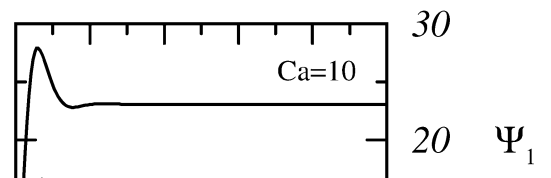

shear strain, $\gamma$ viscosity. For the $\mathrm{Ca}=1$ blend, we report the steadystate viscometric properties for three different droplet concentrations in Fig. 12. The case $\phi=1$ corresponds to the MM Model and it is identical to the stress tensor predictions in $\mathrm{Yu}$ et al. (2002a). For $\mathrm{Ca}=10$, we observe that the power-law index of the steady-state shear viscosity and the first normal stress coefficient are almost insensitive to a variation of $0.005<\theta<0.02$.

Figure 13 is the same as Fig. 12 for the second normal stress coefficient and for the ratio of normal stress coefficients. In the low Capillary Number regime, we note that $\Psi_{2}$ is very small and almost constant over the entire regime of shear rates displayed in Fig. 13. For $\dot{\gamma} \approx 5$, we observe an overshoot in $-\Psi_{2}$ and then the onset of a shear-thinning region. The negative ratio of the normal stress coefficients is small and constant for low values of the coupling parameter. It shows a sudden increase at $\dot{\gamma} \approx 3$ for higher values of the coupling parameter. For equi-viscosity conditions, we report the second normal stress coefficient and the ratio $-\Psi_{2} / \Psi_{1}$ for several droplet concentrations. Over the entire range of droplet concentrations examined, we recover a shearthinning behavior and for vanishing matrix phase, $\phi=1$, we recover the ratio $\Psi_{2} / \Psi_{1}=-0.5$. In the high Capillary Number regime, we recover the first Newtonian plateau for $-\Psi_{2}$ in the low shear-rate region, a power-law behavior for intermediate shear rates, and a second Newtonian plateau for high shear rates. For $\mathrm{Ca}=10$, the power-law index of $-\Psi_{2}$ increases as the coupling parameter is increased.

\section{Elongational flow}

Figure 14 summarizes the predictions of the continuum equations in uniaxial elongational flow, $\nabla_{1} v_{1}=\dot{\epsilon}$, 
Fig. 11 The same as Fig. 10 for the second normal stress coefficient, $\Psi_{2}$ (left column), and the ratio $-\Psi_{2} / \Psi_{1}$ (right column). The dot-dashed horizontal lines in the right column are the steady-state predictions of the MM Model, $\Psi_{2} / \Psi_{1}=-0.5$

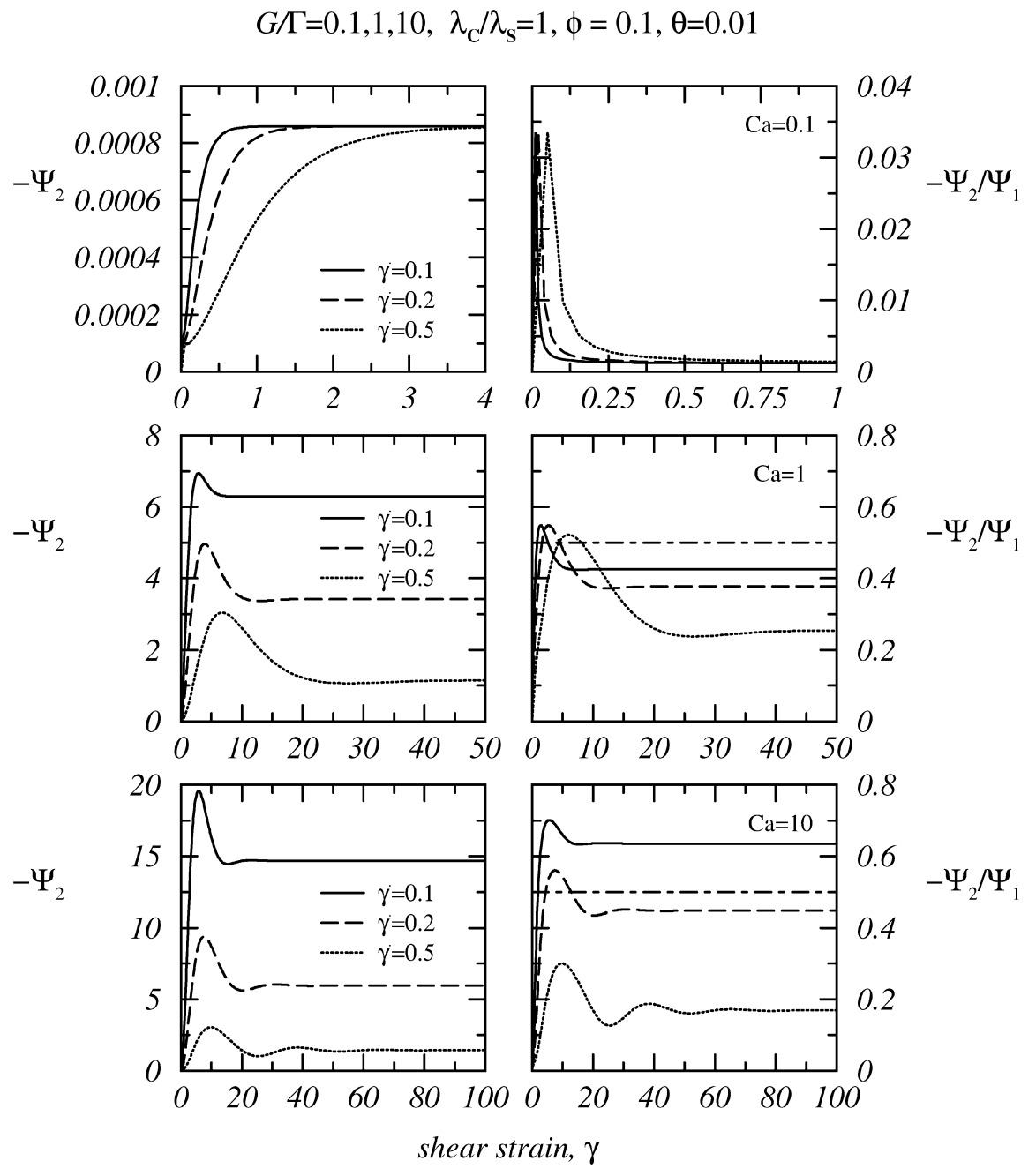


Fig. 12 The steady-state shear viscosity, $\eta_{\mathrm{S}}$ (left column), and the first normal stress coefficient, $\Psi_{1}$ (right column), for three blends $\mathrm{Ca}=0.1$ (first row), 1 (second row), 10 (third row) with dispersed phase concentration, $\phi=0.1$, and three coupling parameter values, $\theta$, denoted in the legend within each row. For the $\mathrm{Ca}=1$ blend, we show three droplet concentrations

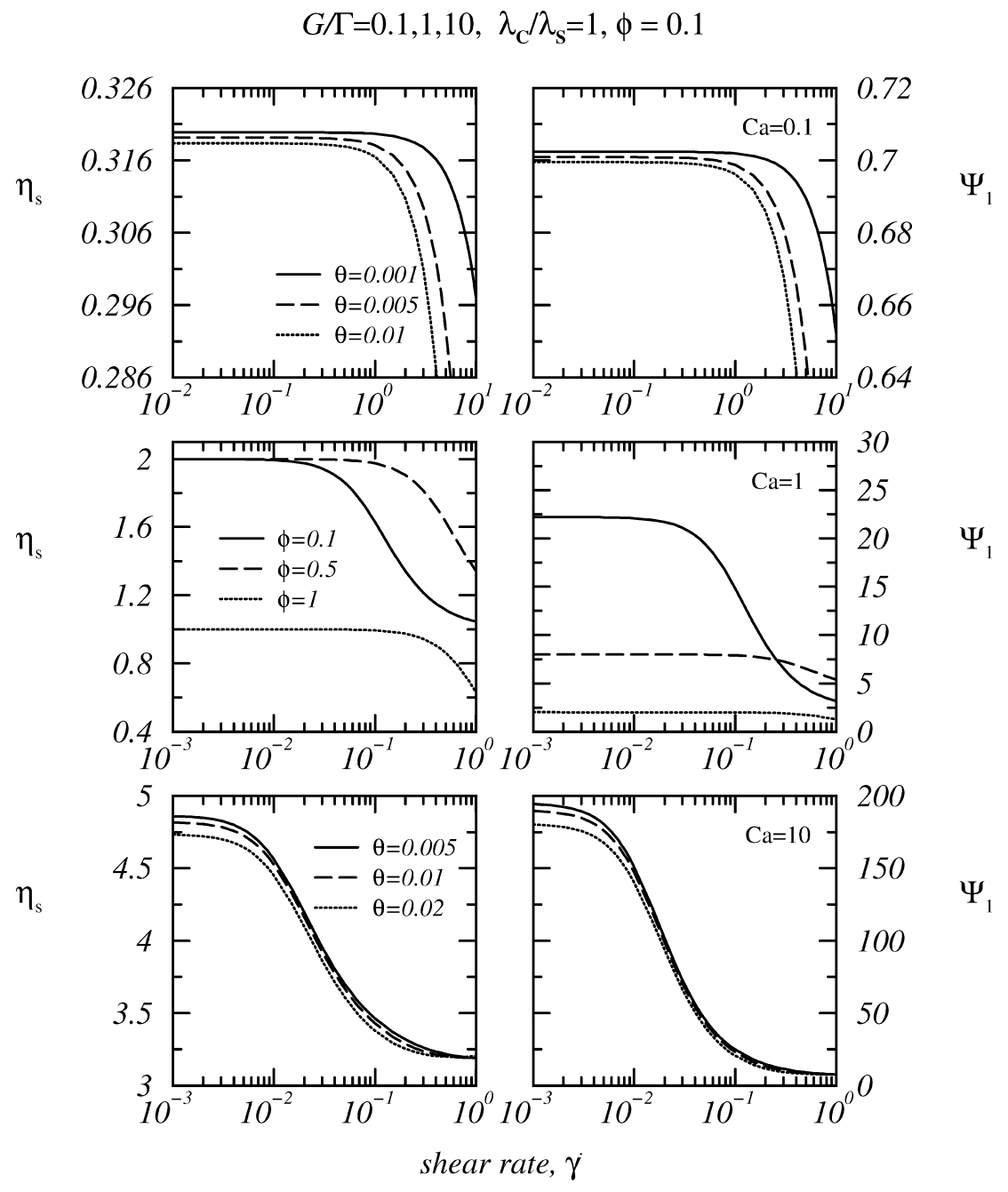

approaches $\dot{\epsilon} \approx 0.1$. The range of coupling parameter values investigated in Fig. 14 has only a minor influence on the steady-state morphological and rheological properties in the high elongation-rate regime.

Figure 15 presents the predictions of the continuum equations for planar elongational flow, $\nabla_{1} v_{1}=\dot{\epsilon}$, $\nabla_{2} v_{2}=-\dot{\epsilon}$, and $\nabla_{3} v_{3}=0$. In the left column of Fig. 15a-c, we plot the transient behavior of the three semiaxes and of the elongational viscosity for two elongation rates. For both elongation rates, we observe a monotonic increase of $L$ and $W$ and a monotonic decrease of $B$ in Fig. 15a,b. This is contrary to the predictions of the MM Model, which predicts prolate droplet configurations in planar elongational flow (see $\mathrm{Yu}$ et al. 2002a, Fig. 10). The transient elongational viscosity in Fig. 15c shows the same qualitative behavior as the transient evolution of $L$ and $W$, and it also increases monotonically. The steady-state morphological and viscometric properties of the blend are reported in Fig. 15d-f for several coupling parameter values. Whereas for uniaxial elongational flow (cf. Fig. 14d,e) we observe a stretching of the droplets into elongated, prolate ellipsoids, the situation is more complicated for planar elongational flow (Fig. 15d,e). In this flow field, the droplets are first stretched into prolates, and in this range of deformation rates the elongational viscosity remains almost constant. However, for higher values of the elongation rate, the droplets start to expand in the 3-direction deforming into an oblate state. The steady-state curves are insensitive with respect to a variation of $\theta$ between 0.01 and 0.07 . The equations predict a strong strain hardening of the blend being subjected to planar hyperbolic flow. The beginning of the strain hardening regime (Fig. 15f) is observed in a rather narrow range of elongation rates, and it correlates with a sharp increase of the $W$-axis which is observed in the same elongationrate interval (Fig. 15e). The major semiaxis, $L$, and the minor semiaxis, $B$, in Fig. $15 \mathrm{~d}$,e show a smooth increase and a smooth decay to zero, respectively. 
Fig. 13 The same as Fig. 12 for the steady-state second normal stress coefficient, $\Psi_{2}$ (left column), and the ratio $-\Psi_{2} / \Psi_{1}$ (right column). The horizontal lines in the right column are the predictions of the MM Model, $\Psi_{2} / \Psi_{1}=-0.5$

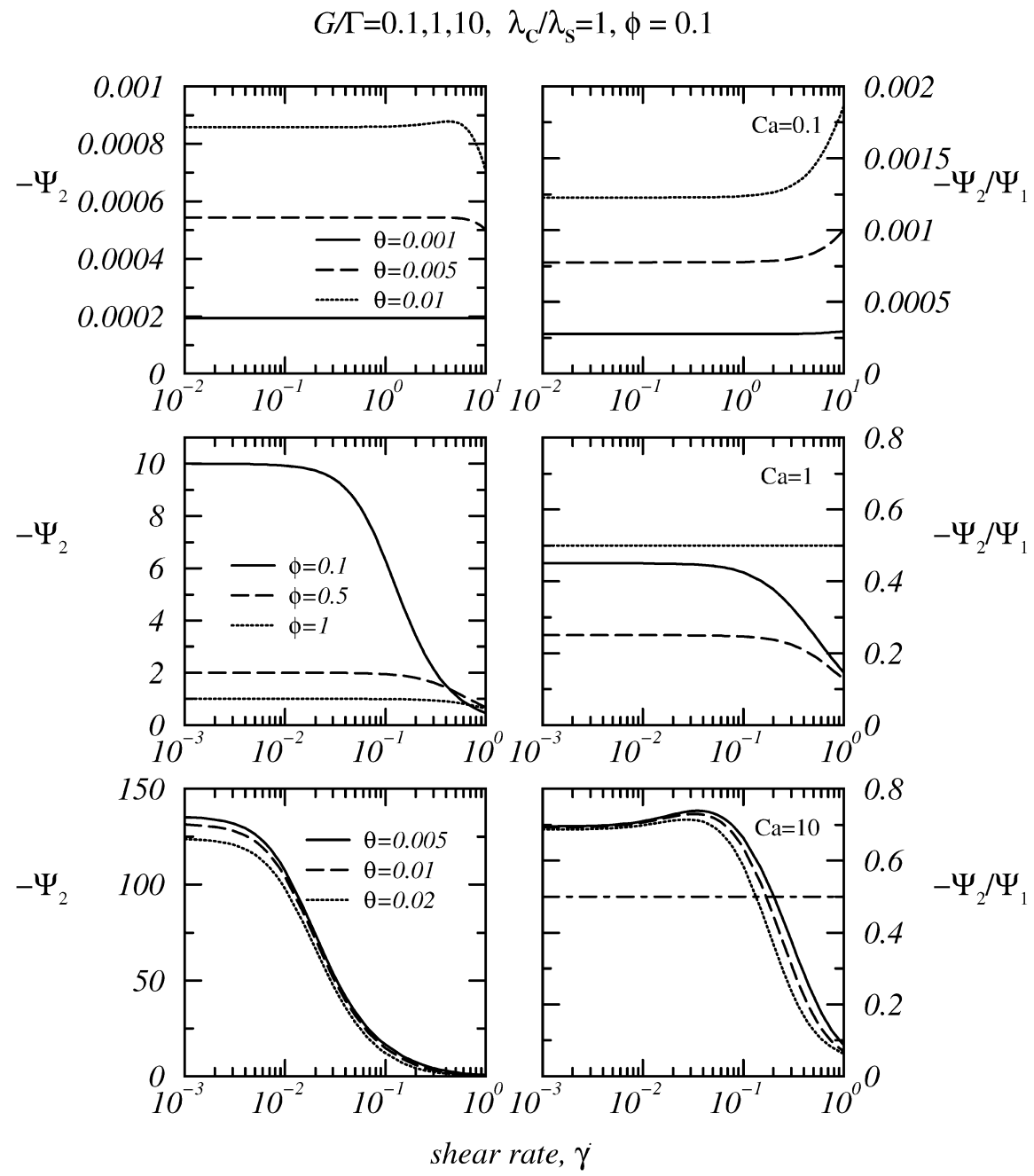

Therefore, in the present situation, the strain hardening in planar elongational flow is related to an expansion of the droplets into the 3 -direction. Note that the strain hardening behavior recovered here is a feature which was not observed in the recent work of $\mathrm{Yu}$ et al. (2002a), who evaluated the extra stress tensor for the MM model. Yu et al. (2002a) report a constant elongational viscosity in uniaxial elongational flow and in planar elongational flow (see Yu et al. 2002a, Eqs. 40, 44 and Figs. 8, 10). Viscometric properties of the ALS model in uniaxial elongational flow have not been reported thus far. Further sample calculations in planar elongational flow are shown in Fig. 17, which will be presented in the following subsection.

Four-roll mill flow

We have solved the time evolution equations for a deformation that is found at the stagnation point of the four roll mill: $\nabla v=\frac{1}{2} g\left(\begin{array}{ccc}1+\alpha & 1-\alpha & 0 \\ -1+\alpha & -1-\alpha & 0 \\ 0 & 0 & 0\end{array}\right)$

where $g$ is the strength of the deformation field, and $-1<\alpha<1$, is a measure of the relative strength of the straining motion and the vorticity in the flow. A purely rotational flow, a purely straining motion, and a simple shear flow are obtained with $\alpha=-1,+1$, and 0 , respectively.

In Fig. 16 we display the droplet semiaxes and the droplet orientation angle for two model blends with $\mathrm{Ca}=0.25$ (Fig. 16a) and $\mathrm{Ca}=4$ (Fig. 16b) for start-up of a four-roll mill flow. We chose $\alpha=0.2$, i.e., the magnitude of strain rate, $1+\alpha$, over the magnitude of vorticity, $1-\alpha$, is three halves. The $\mathrm{Ca}=0.25$ and the $\mathrm{Ca}=4$ blend are investigated for high and low values of the velocity gradient strength, $g$, respectively. We have chosen the same droplet concentration and coupling parameter value for both blends, and we have tracked the transient 
Fig. 14 The morphological and viscometric properties of the $\mathrm{Ca}=4$ blend with $\phi=0.1$ dispersed phase concentration in uniaxial elongational flow: $(a-c)$ the transient behavior of the droplet semiaxes and the elongational viscosity for three elongation rates, $\dot{\epsilon}=0.1$ (solid lines), 0.2 (dashed lines), 0.4 (dotted lines), and $\theta=0.1 ;(\mathrm{d}-\mathrm{f})$ the steady-state properties of the same quantities for three coupling parameter values, $\theta=0.05$ (solid lines), 0.1 (dashed lines), 0.2 (dotted lines)

$$
G / \Gamma=4, \lambda_{\mathrm{c}} / \lambda_{\mathrm{s}}=1, \phi=0.1, \theta=0.1
$$
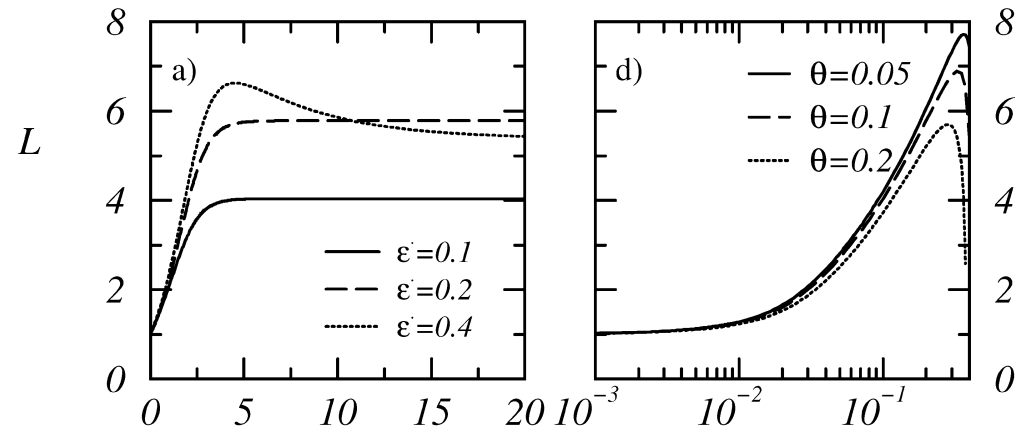

$L$
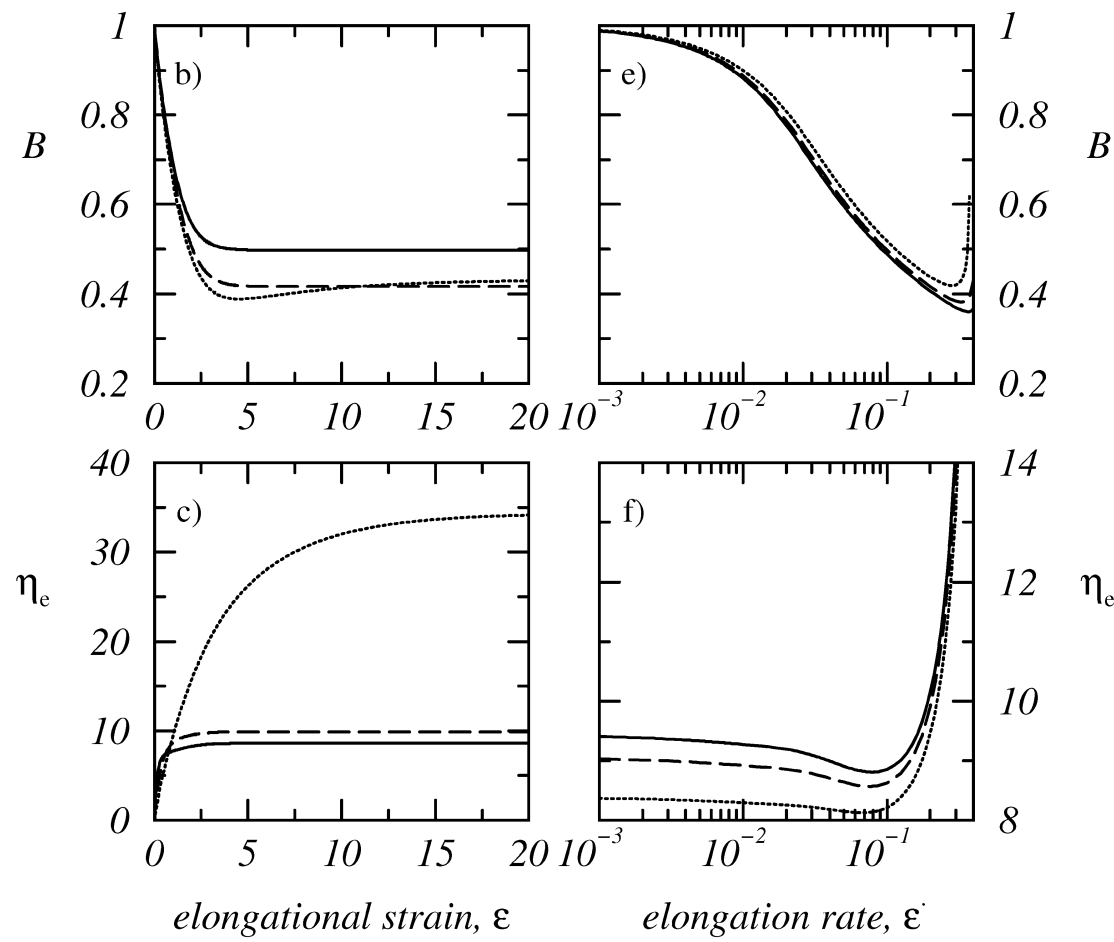

droplet morphology for three strengths of the deformation field. For the $\mathrm{Ca}=0.25$ blend in Fig. 16a, we observe a monotonic behavior of the droplet semiaxes for small times and a steady state is obtained after a dimensionless time of approximately one decade. For this Capillary Number and the rather high values for $g$, oblate droplet configurations are generated in the fourroll mill. The orientation angle shows a non-linear increase with a pronounced overshoot. The qualitative behavior is different for the $\mathrm{Ca}=4$ model blend and the rather low values of $g$ (Fig. 16b), where a non-monotonic approach with damped oscillations is observed for the transient behavior of the droplet morphology. A steady state is attained at long times. In general, such an oscillatory behavior of an internal variable is observed if a corotational derivative is employed for the time evolution equations of the structural variable. Here, the oscillations in the droplet shape tensor components are due to the non-linear coupling of the time evolution equations involving the structural variables. For $\mathrm{Ca}=4$, we observe a competition between oblate and prolate droplet shapes for small times immediately after start-up of flow. In the steady state, a prolate droplet configuration is recovered. The orientation angle shows an increase with dimensionless time.

Figure 17 displays the steady state Taylor Deformation Parameter, $D=(L-B) /(L+B)$ for a purely straining motion, $\alpha=1$, and a four-roll mill flow with $\alpha=0.2$ for four different Capillary Numbers and fixed droplet concentration and coupling parameter. Normally, such a representation of the Taylor Deformation Parameter is employed to evaluate rheological models that include 
Fig. 15 The same as Fig. 14 for planar elongational flow: $(a-c)$ the transient behavior of the droplet semiaxes and the elongational viscosity for two elongation rates $\dot{\epsilon}=0.05$ (solid lines), 0.1 (dashed lines), and $\theta=0.01 ;(\mathrm{d}-\mathrm{f})$ the steady-state properties of the same quantities for three coupling parameter values, $\theta=0.01$ (solid lines), 0.05 (dashed lines), 0.07 (dotted lines)

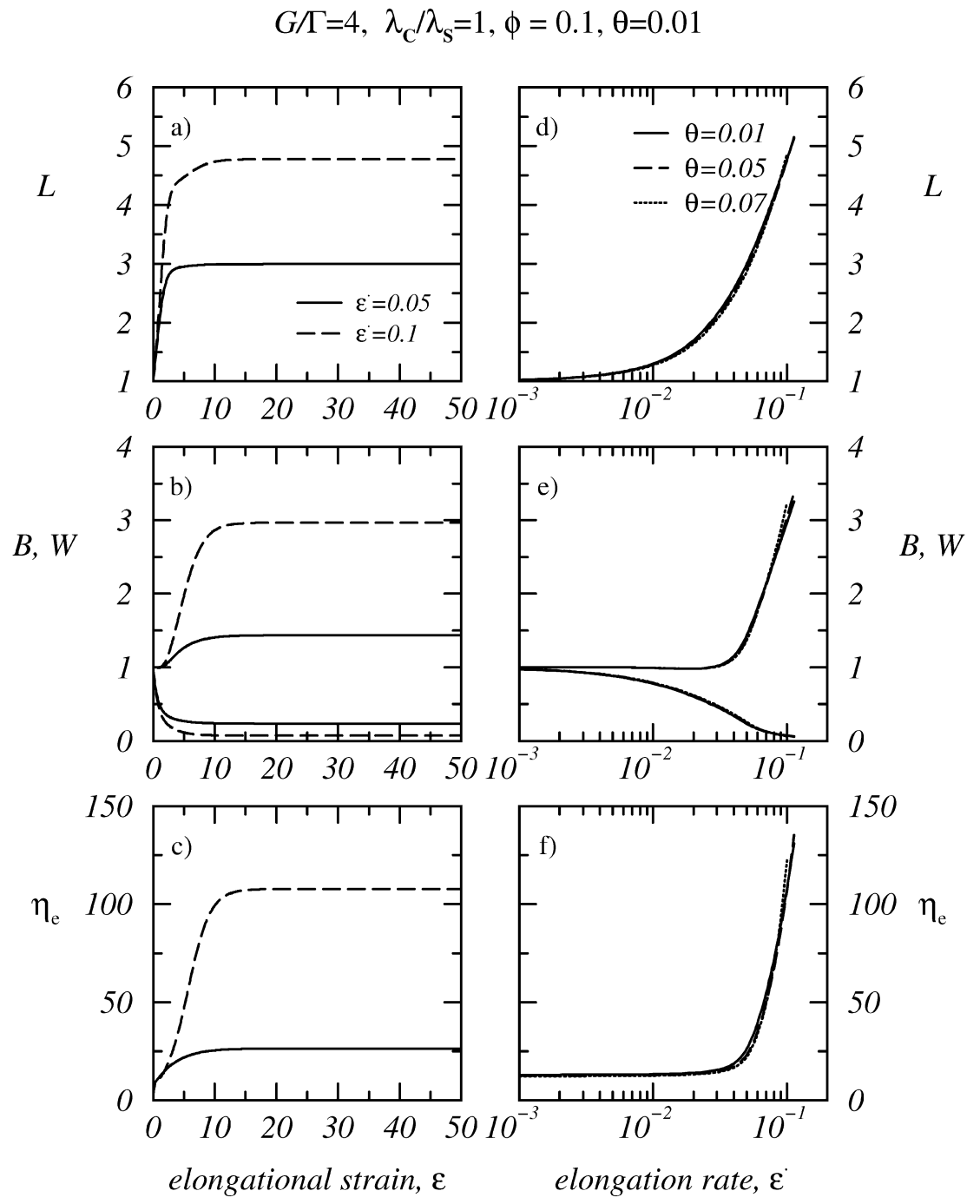


Fig. 16 The transient morphological properties for (a) a $\mathrm{Ca}=0.25$; (b) a $\mathrm{Ca}=4$ model blend for start-up of a four-roll mill flow with $\alpha=0.2$ and three values for the strength of the flow field, $g$. The droplet concentration and the coupling parameter are $\phi=0.1$ and $\theta=0.01$, respectively
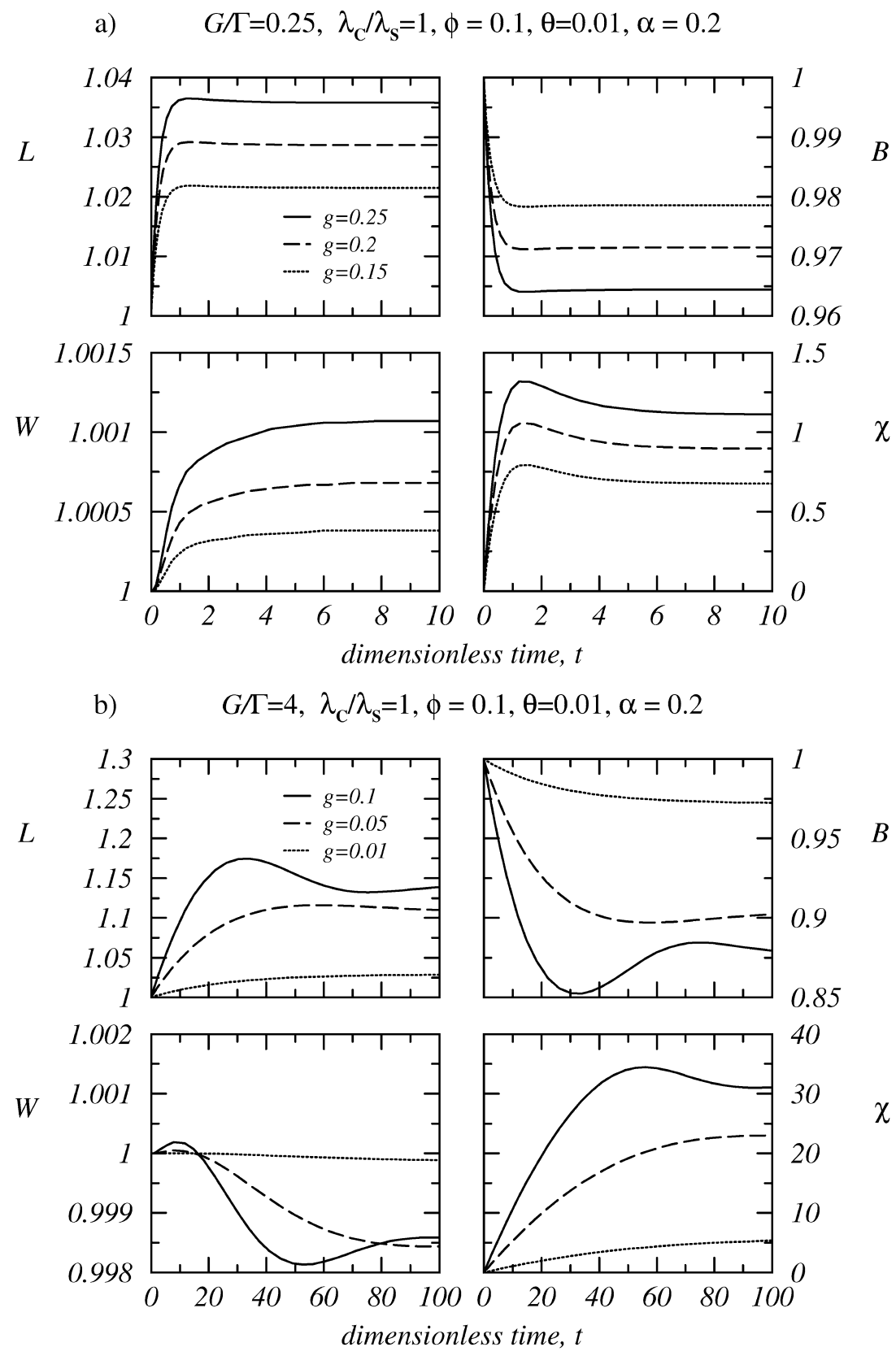

increase and decrease of the major and the minor droplet axes in the shearing plane, respectively. Figure 18b displays a strong decrease of the steady-state orientation angle. Solid lines in Fig. 18 have been calculated for the following values of the ratio of matrix and interfacial elasticity, the viscosity ratio, and the droplet concentration: $G / \Gamma=0.13, p=-1.3, \phi=0.01$. The EWM power law index, $k$, has been taken as vanishing. Note that for the calculations in Fig. 18 the phenomenological coefficient $p$ has been taken as the viscosity ratio of the blend components, i.e., $p=-\eta^{*} / \eta_{\mathrm{c}}=-1.3$ is an independent quantity. For the purpose of the present comparison with experimental data we chose $\phi=0.01$ which is reasonable to represent the low concentration regime of a single droplet experiment. To fit experimental data the following values for the relaxation times and the phenomenological coupling parameter have been adopted: $\lambda_{\mathrm{c}}=10^{-3} \mathrm{~s}, \lambda_{\mathrm{s}}=5 \times 10^{-4} \mathrm{~s}, \theta=0.01$. (Note that model predictions are insensitive for $0.01<\theta<0.05$.) We notice that the fit of the droplet semiaxes is satisfactory in Fig. 18, whereas the strong decrease of the orientation angle is not predicted by our model. The strong decrease 
Fig. 17 The Taylor Deformation Parameter, $D$, as a function of the magnitude of the velocity gradient at the stagnation point of a four-roll mill flow for four Capillary Numbers $\mathrm{Ca}=10,1$, $0.5,0.25$, and constant droplet concentration, $\phi=0.1$, and $\theta=0.01$. Two examples of the strength of the straining motion are shown: $\alpha=1$ (solid lines) and $\alpha=0.2$ (dashed lines)

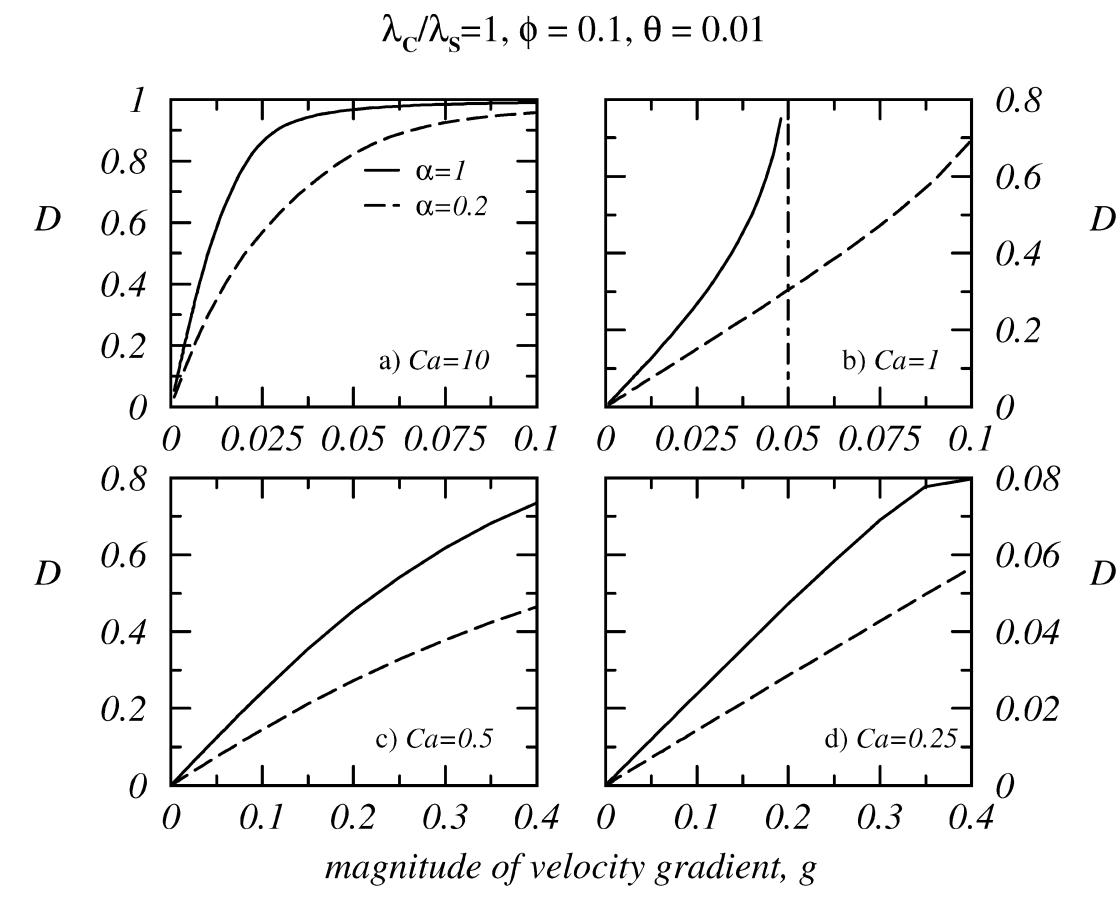

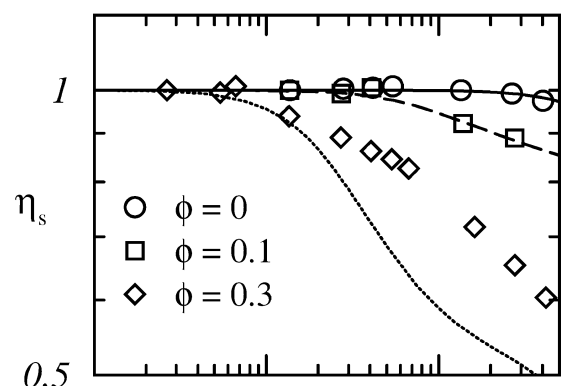

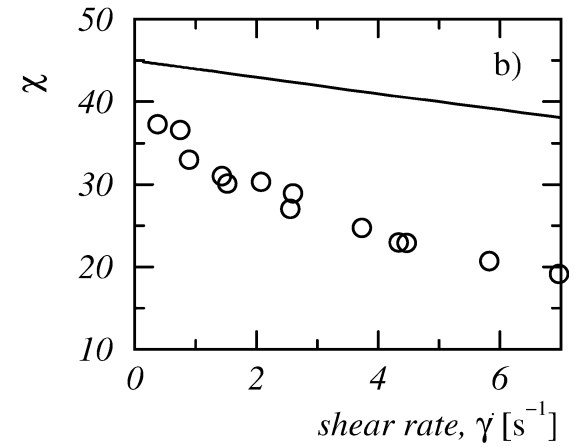

Fig. 18 The: (a) major and the minor semiaxes, $L, B$; (b) orientation angle, $\chi$ of a single silicon droplet in a viscoelastic corn syrup matrix (symbols) together with model predictions (solid lines). Experimental data are from Guido et al. (2003)

of the orientation angle with increasing shear rate is presumably due to a non-affine motion of the silicon droplet with respect to the externally-imposed flow field and it may be recovered using the Gordon-Schowalter

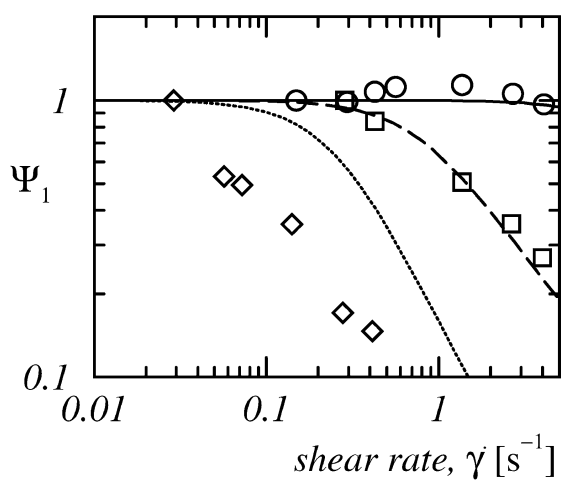

Fig. 19 The steady-shear viscosity and the first normal stress coefficient for pure PDMS (circles) and two PIB/PDMS blends (squares, diamonds) together with model predictions (lines). Experimental data are from Vinckier et al. (1996)

derivative in the droplet shape tensor, Eq. (4c). The perturbative approach proposed by Greco (2002) gives a more satisfactory fit to experimental data compared to the present model - cf. Guido et al. (2003) (Fig. 4). 
Vinckier et al. (1996) studied morphological and rheological properties of different blends of polyisobutene (PIB, $\eta^{*}=86 \mathrm{~Pa}$ s) and polydimethylsiloxane $\left(\mathrm{PDMS}, \eta_{\mathrm{c}}=195 \mathrm{~Pa} \mathrm{~s}, \Psi_{1, \mathrm{c}}=10.4 \mathrm{~Pa} \mathrm{~s}^{2}\right.$ ) in steady shear flow. Whereas pure PDMS shows a pronounced shear thinning behavior, pure PIB is Newtonian for shear rates $\dot{\gamma}<10 \mathrm{~s}^{-1}$ (Vinckier et al. 1996). Therefore, we compare experiments on PIB/PDMS blends with our model. Interfacial tension between the blend components is $\Gamma^{\prime}=2.3 \mathrm{mN} / \mathrm{m}$. With the UCMM and the relationshipfor the interfacial elastic modulus we obtain $G \approx 7300 \mathrm{~Pa}$ and $\Gamma=230 \mathrm{~Pa}$ for an average droplet radius of $10 \mu \mathrm{m}$. In what follows, we fit rheological measurements of $10 \mathrm{wt} \%$ and $30 \mathrm{wt} \%$ PIB in PDMS with our model.

In Fig. 19, we show the steady-state shear viscosity and the first normal stress coefficient for the viscoelastic PDMS matrix fluid and for two different PIB/PDMS blends (symbols) together with model predictions (lines). Experimental data have been taken from Vinckier et al. (1996) and they have been normalized with their zero shear rate values. Circles in Fig. 19 are for the viscometric properties of the pure PDMS matrix fluid, squares and diamonds represent the $\phi=0.1$ and the $\phi$ $=0.3 \mathrm{PIB} / \mathrm{PDMS}$ blend, respectively. Solid lines in Fig. 19 have been calculated from the model equations with $G=7300 \mathrm{~Pa}, \phi=0$, and $k=-2$ for the EWM power law index to fit the shear thinning of the matrix phase. The dashed lines and the dotted lines in Fig. 19 have been calculated for $G / \Gamma=30, k=-2$ and $\phi=0.1,0.3$ for the $10 \mathrm{wt} \%$ and the $30 \mathrm{wt} \%$ blends, respectively. The relaxation times and the phenomenological coupling parameter have been chosen to recover the shear-thinning behavior. This is reasonable since there is no standard technique available to determine the relaxation time of the interface, $\lambda_{\mathrm{s}}-\mathrm{cf}$. Vinckier et al. (1996) (Figs. 6 and 7). We took $\lambda_{\mathrm{c}}=0.03 \mathrm{~s}, \theta=0$ for pure PDMS, $\lambda_{\mathrm{c}} / \lambda_{\mathrm{s}}=0.012, \theta=0.01$ for the $\phi=0.1$ blend, and $\lambda_{\mathrm{c}} / \lambda_{\mathrm{s}}=0.04, \theta=0.01$ for the $\phi=0.3$ blend. Note that in Fig. 19 the phenomenological coefficient $p$ is related to the elasticity of the matrix phase and the interface, i.e., $p=-\left(\Gamma \lambda_{\mathrm{s}}\right) /\left(G \lambda_{\mathrm{c}}\right)$. We note that model predictions for the steady-shear viscosity and the first normal stress coefficient are satisfactory for $\phi=0.1$. For $\phi=0.3$ only the onset of the shear-thinning behavior is recovered from the model and the model fails to predict the quantitative behavior of the first normal stress coefficient. The fit to both rheological characteristic functions is satisfactory only in the dilute regime, $\phi<0.2$.

\section{Conclusions}

A set of time evolution equations of polymer blends with droplet morphology has been developed in the framework of non-equilibrium thermodynamics taking into account matrix phase viscoelasticity. Our description is restricted to blends without any droplet breakup or aggregation, viscoelastic material properties are present only in the matrix phase but not in the droplet phase, and the equations derived herein apply to dilute blends since droplet-droplet interactions have not been incorporated into the modeling. We have adopted two microstructural variables that take into account the conformation of the matrix molecules and the ellipsoidal shape of the volume-preserving micro-droplets. The droplet shape tensor used herein has a direct connection to the underlying droplet volume, in contrast to the anisotropy tensor adopted by Almusallam et al. (2000). Moreover, it is possible to recover prolate as well as oblate droplet shapes from the system equations (cf. Maffettone and Minale 1998) and the droplet configuration equation does not involve the Gordon-Schowalter derivative. Finally, a strain-hardening effect can be predicted from our continuum equations which was not recovered in the previous work of Yu et al. (2002a) who obtained a constant elongational viscosity.

Sample calculations have been carried out to study the transient and steady-state blend properties in various flows, such as start-up of simple shear flow, uniaxial elongational flow, planar elongational flow, and the four-roll mill flow. Our calculations in steady-shear flow yield a compression of the major droplet axis and a simultaneous droplet widening for high shear rates. This morphological behavior correlates with the typical nonlinear viscoelastic response of synthetic polymeric materials. For uniaxial elongational flow we recover a strain softening for intermediate elongation rates and a strain hardening for higher elongation rates. In planar elongational flow, the strain hardening correlates with a droplet widening in the neutral direction. For start-up of the four-roll mill flow, we observe oscillations of the droplet axes that have not been observed to date in experiments. A fit of the model to experimental data of Guido et al. (2003) and Vinckier et al. (1996) has been presented.

Acknowledgements BJE acknowledges financial support provided by ETH-Zürich and The University of Tennessee. MD acknowledges Prof. P. L. Maffettone for fruitful discussions. 


\section{References}

Aït-Kadi A, Ramazani A, Grmela M, Zhou C (1999) Volume preserving rheological models for polymer melts and solutions using the GENERIC formalism. J Rheol 43:51-72

Almusallam AS, Larson RG, Solomon MJ (2000) A constitutive model for the prediction of ellipsoidal droplet shapes and stresses in immiscible blends. J Rheol 44:1055-1083

Beris AN, Edwards BJ (1990a) Poisson bracket formulation of incompressible flow equations in continuum mechanics. J Rheol 34:55-78

Beris AN, Edwards BJ (1990b) Poisson bracket formulation of viscoelastic flow equations of differential type-a unified approach. J Rheol 34:503-538

Beris AN, Edwards BJ (1994) Thermodynamics of flowing systems. Oxford University Press, New York

Doi M, Ohta T (1991) Dynamics and rheology of complex interfaces. J Chem Phys 95:1242-1248

Edwards BJ, Beris AN (1991a) Noncanonical Poisson bracket for nonlinear elasticity with extensions to viscoelasticity. J Phys A Math Gen 24:2461-2480

Edwards BJ, Beris AN (1991b) Unified view of transport phenomena based on the generalized bracket formulation. Ind Eng Chem Res 30:873-881

Edwards BJ, Dressler M (2003) A rheological model with constant approximative volume for immiscible blends of ellipsoidal droplets. Rheol Acta 42:326337

Edwards BJ, Beris AN, Grmela M (1991) The dynamic behavior of liquid crystals - a continuum description through generalized brackets. Mol Cryst Liquid Cryst 201:51-86

Edwards BJ, Beris AN, Mavrantzas VG (1996) A model with two coupled Maxwell modes. J Rheol 40:917-942

Edwards BJ, Dressler M, Grmela M, AïtKadi A (2003) Rheological models with microstructural constraints. Rheol Acta 42:64-72
Greco F (2002) Drop deformation for nonNewtonian fluids in slow flows. J NonNewtonian Fluid Mech 107:111-131

Grmela M (1988) Hamiltonian dynamics of incompressible elastic fluids. Phys Lett A 130:81-86

Grmela M (1989) Hamiltonian mechanics of complex fluids. J Phys A Math Gen 22:4375-4394

Grmela M, Aït-Kadi A (1998) Rheology of inhomogeneous immiscible blends. J Non-Newtonian Fluid Mech 77:191199

Grmela M, Carreau PJ (1987) Conformation tensor rheological models. J NonNewtonian Fluid Mech 23:271-294

Grmela M, Öttinger HC (1997) Dynamics and thermodynamics of complex fluids. I. Development of a general formalism. Phys Rev E 56:6620-6632

Grmela M, Aït-Kadi A, Utracki LA (1998) Blends of two immiscible and rheologically different fluids. J Non-Newtonian Fluid Mech 77:253-259

Grmela M, Bousmina M, Palierne JF (2001) On the rheology of immiscible blends. Rheol Acta 40:560-569

Guido S, Villone M (1998) Three-dimensional shape of a drop under simple shear flow. J Rheol 42:395-415

Guido S, Simeone M, Greco F (2003) Effects of matrix viscoelasticity on drop deformation in dilute polymer blends under slow shear flow. Polymer 44:467471

Hu YT, Lips A (2003) Transient and steady state three-dimensional drop shapes and dimensions under planar extensional flow. J Rheol 47:349-369

Jansseune T, Mewis J, Moldenaers P, Minale M, Maffettone PL (2000) Rheology and rheological morphology determination in immiscible two-phase polymer model blends. J Non-Newtonian Fluid Mech 93:153-165

Jansseune T, Vinckier I, Moldenaers P, Mewis J (2001) Transient stresses in immiscible model polymer blends during start-up flows. J Non-Newtonian Fluid Mech 99:167-181
Lacroix C, Grmela M, Carreau PJ (1998) Relationships between rheology and morphology for immiscible molten blends of polypropylene and ethylene copolymers under shear flow. J Rheol 42:41-62

Levitt L, Macosko CW, Pearson SD (1996) Influence of normal stress difference on polymer drop deformation. Polym Eng Sci 36:1647-1665

Lhuillier D (2003) Dynamics of interfaces and rheology of immiscible liquid-liquid mixtures. C R Mecanique 331:113-118

Maffettone PL, Minale M (1998) Equation of change for ellipsoidal drops in viscous flow. Erratum (1999) 84:105-106. J Non-Newtonian Fluid Mech 78:227241

Minale M, Maffettone PL (2003) Morphology estimation from normal stress measurements for dilute immiscible polymer blends. Rheol Acta 42:158-165

Öttinger HC, Grmela M (1997) Dynamics and thermodynamics of complex fluids. II. Illustrations of a general formalism. Phys Rev E 56:6633-6655

Souvaliotis A, Beris AN (1992) An extended White-Metzner viscoelastic fluid model based on an internal structural parameter. J Rheol 36:241-271

Vinckier I, Moldenaers P, Mewis J (1996) Relationship between rheology and morphology of model blends in steady shear flow. J Rheol 40:613-631

Wagner NJ, Öttinger HC, Edwards BJ (1999) Generalized Doi-Ohta model for multiphase flow developed via GENERIC. AICHE J 45:1169-1181

$\mathrm{Yu} \mathrm{W}$, Bousmina M, Grmela M, Palierne JF, Zhou CX (2002a) Quantitative relationship between rheology and morphology in emulsions. J Rheol 46:1381-1399

$\mathrm{Yu}$ W, Bousmina M, Grmela M, Zhou CX (2002b) Modeling of oscillatory shear flow of emulsions under small and large deformation fields. J Rheol 46:14011418 